\title{
Roland Barthes : genèse d'un séminaire inédit
}

\section{Présenté par Maria O’Sullivan}

\section{OpenEdition}

\section{Journals}

Édition électronique

URL : http://journals.openedition.org/genesis/1413

DOI : 10.4000/genesis. 1413

ISSN : 2268-1590

\section{Éditeur :}

Presses universitaires de Paris Sorbonne (PUPS), Société internationale de génétique artistique littéraire et scientifique (SIGALES)

\section{Édition imprimée}

Date de publication : 17 novembre 2014

Pagination : 133-164

ISBN : 9782840509714

ISSN : 1167-5101

Référence électronique

Présenté par Maria O'Sullivan, «Roland Barthes : genèse d'un séminaire inédit », Genesis [En ligne], 39 | 2014, mis en ligne le 05 janvier 2017, consulté le 01 mai 2019. URL : http:// journals.openedition.org/genesis/1413; DOI : 10.4000/genesis.1413 


\title{
Roland Barthes : genèse d'un séminaire inédit
}

\author{
Présenté par Maria O’Sullivan
}

$\mathrm{E}$ n octobre 1966, Roland Barthes est, depuis déjà quatre ans, maître de conférences à l'École pratique des hautes études. Après deux années de séminaire consacrées à l'analyse sémiologique, puis deux années de travail sur la rhétorique, il choisit pour nouveau sujet «le discours historique ». Ce séminaire de 1966-1967 se compose en fait de deux sections : « La linguistique du discours » (du 10 novembre 1966 jusqu'à une date inconnue de janvier 1967) et "Le discours de l'histoire » (du 3 février au 2 juin 1967). Les notes rédigées à cette occasion sont aujourd'hui conservées dans le fonds Barthes du département des Manuscrits de la Bibliothèque nationale de France.

Les feuillets dont il sera ici question ont servi de support à deux séances de ce séminaire : la quatrième, celle du 16 décembre 1966 ( « Le discours, rémunération de la langue », f. 23-33), et la cinquième, qui s'est tenue à une date incertaine de janvier 1967 («L'espace du langage (le paragrammatisme) », f. 34-45)1. Ces feuillets n'ont abouti à aucune publication du vivant de Barthes ; leur contenu est resté inconnu jusqu'à ce jour. Ceux qui précèdent sont, en revanche, proches de l'article «Écrire, verbe intransitif ? ( $\left.1970^{2}\right)$, ceux qui suivent, de l'article «Le discours de l'histoire » (19673). Or, ces notes de décembre 1966 et janvier 1967 sont d'un intérêt majeur pour la compréhension de toute la suite de l'œuvre de Barthes. On y découvre notamment les toutes premières traces de l'influence que la pensée de Jacques Derrida et celle de Julia Kristeva exerceront sur sa théorisation, mais aussi sur sa pratique à venir de l'écriture. Barthes a bien sûr explicitement reconnu l'influence de Kristeva (notamment dans « L'Étrangère » en 19704) : arrivée à Paris en 1965, elle était alors une des étudiantes du séminaire et lui communiqua plusieurs de ses textes encore inédits ${ }^{5}$. Le nom de Derrida, lui, a toujours été rare sous la plume de Barthes. Bien que le jeune philosophe ait publié quelques articles dans Critique ou dans d'autres revues dès le début des années soixante ${ }^{6}$, Barthes ne semble pas avoir connu son travail avant de le rencontrer au grand colloque structuraliste qui s'est tenu à l'université Johns Hopkins de Baltimore en octobre 19667 .

\section{Séance de janvier 1967}

À l'ouverture de la cinquième séance dont nous donnons ici les notes, Barthes affirme que tout ce qui a précédé ne formait qu'une "longue introduction sur la linguistique du discours » (f. 34, fig. 1), fondée sur une conception classique du langage et une définition

1. Pour des raisons de place, l'édition papier de ce numéro de Genesis ne présente que la cinquième séance. En revanche, l'édition électronique contiendra la présentation, le fac-similé et la transcription de la quatrième et de la cinquième séance.

2. Roland Barthes, Euvres complètes (désormais $O C$ ), éd. Éric Marty, Paris, Éditions du Seuil, 2002, t. III, p. 617-626.

3. $O C$, t. II, p. $1250-1262$.

4. $O C$, t. III, p. 477-480.

5. Voir Julia Kristeva, « Mémoire », L'Infini, n 1, hiver 1983, p. 39-54, et Les Samouraïs, Paris, Fayard, 1990.

6. Notamment : «De la grammatologie I », Critique, $\mathrm{n}^{\circ} 223$, décembre 1965, p. 1016-1042; « De la grammatologie II », Critique, $\mathrm{n}^{\circ} 224$, janvier 1966 , p. 23-53 ; «Freud et la scène de l'écriture », Tel Quel, n 26, été 1966, p. 10-41.

7. Voir Jacques Derrida, «La structure, le signe et le jeu dans le discours des sciences humaines », publié dans L'Écriture et la différence, Paris, Éditions du Seuil, 1967, p. 409-428. 
linéaire de la parole. Il manifeste alors son intention de s'intéresser à la profondeur du discours et de « rappeler qu'il est un espace (et non une ligne) ». Pour mieux comprendre cette déclaration, il faut d'abord s'arrêter sur la première des deux références à Roman Jakobson qui apparaissent ici. Barthes affirme en effet que Jakobson « aplatit le paradigme [...] en syntagme » (f. 36), en songeant à un passage de « Linguistique et poétique » (1960), où Jakobson déclare que « la fonction poétique projette le principe d'équivalence de l'axe de la sélection sur l'axe de la combinaison ${ }^{8}$ ». Dans le discours littéraire, les séquences de mots entretiendraient ainsi des rapports d'équivalence, de similarité ou de différence avec celles qui les précèdent et les suivent. Les phénomènes d'assonance et d'allitération, exemples privilégiés de Jakobson, attestent que l'apparition des sons fait attendre le retour de sons similaires dans la suite de la séquence. On est proche ici de l'idée de rémunération développée par Barthes lors du séminaire du 16 décembre 1966, mais on reste encore dans une conception linéaire du langage.

Pour penser un autre modèle, plus spatial cette fois, Barthes utilise des textes encore inédits de Julia Kristeva9 ${ }^{9}$ S'autorisant de celle-ci, Barthes donne tout d'abord une rapide présentation du dialogisme bakhtinien et explique ce que sont les « anagrammes » de Saussure. Ces derniers venaient en effet d'être découverts dans un fonds d'archives que des chercheurs comme Robert Godel, Émile Benveniste et Jean Starobinski ont mis au jour et commenté. Saussure y montre comment, par un jeu de retour des mêmes phonèmes, les vers saturniens latins renvoient en fait à des mots ou à des noms qui n'apparaissent jamais à la surface du texte. Or, dans «Pour une sémiologie des paragrammes », Kristeva se sert de cette idée d'anagramme ou de paragramme (terme plus juste que celui de Saussure) pour envisager un modèle linguistique et critique nouveau :

Nous appelons réseau paragrammatique le modèle tabulaire (non linéaire) de l'élaboration de l'image littéraire, autrement dit, le graphisme dynamique et spatial désignant la pluridétermination du sens [...] dans le langage poétique ${ }^{10}$.

Pour Kristeva, les paragrammes de Saussure permettent en effet de penser une dimension fondatrice du texte littéraire : "Ainsi dans le paragramme d'un texte fonctionnent tous les textes de l'espace lu par l'écrivain11. »Elle met cela en relation avec l'idée d' « intertextualité », dont elle trouve les fondements théoriques dans l'œuvre de Mikhaill Bakhtine, qu'elle va bientôt faire connaître en France, notamment grâce au texte dont il est question dans cette séance : «Bakhtine, le mot, le dialogue et le roman ». Bakhtine insiste en effet sur la multiplicité des voix et des sens qui réside dans chaque texte, voire derrière chaque mot. Tout comme les paragrammes de Saussure, le dialogisme bakhtinien met en évidence cet instant où l'on s'aperçoit des multiples directions sémantiques et discursives vers lesquelles pointe un texte : « le dialogue n'est pas seulement le langage assumé par le sujet, c'est une écriture où on lit l'autre 12 ».

Le séminaire résume ces positions de Kristeva et exploite les images de profondeur et d'espace textuels qui y sont mobilisées : le nom anagrammatique qui émerge dans la lecture de Saussure est en effet « inscrit dans le vers sous une forme éparse, séparée, espacée » (f. 33) ; Saussure « restitue et maintient une "profondeur" du langage [...] L'espace du langage reste en quelque sorte tangible » (f. 36, fig. 3). Barthes s'accorde avec Kristeva pour dire que l'écriture paragrammatique remplace un modèle linéaire par un modèle plus spatial, et met en relation l'écriture paragrammatique avec un texte de Derrida, « De la grammatologie II » (Critique, $\mathrm{n}^{\circ} 224$, janvier 1966), qui appelle également à l'abandon du modèle linéaire et permet de rendre compte du phénomène de lecture dédoublée que le paragrammatisme met en jeu. Il prend l'œuvre de Sade (il y reviendra dans Sade, Fourier, Loyola en 1971) pour exemple d'une « écriture qui dialogue avec une autre écriture », dans

8. Roman Jakobson, Essais de linguistique générale : les fondations du langage, trad. Nicolas Ruwet, Paris, Minuit, 1963, p. 220.

9. «Pour une sémiologie des paragrammes » (Tel Quel, $n^{\circ} 29$, printemps 1967) ; «Bakhtine, le mot, le dialogue et le roman » (Critique, $\mathrm{n}^{\circ} 239$, avril 1967); «Le texte clos » (Langages, $n^{\circ} 12$, décembre 1968).

10. Julia Kristeva, «Pour une sémiologie des paragrammes », dans Sêmeiôtiké. Recherches pour une sémanalyse, Paris, Éditions du Seuil, 1969, rééd. Points, 1978, p. 123.

11. Ibid., p. 120.

12. J. Kristeva, «Bakhtine, le mot, le dialogue et le roman », art. cit., p. 88. 
la mesure où l' " écriture "érotique" entre sans cesse en dialogue avec des écritures littéraires, philosophiques, lyriques, aphoristiques, mythologiques » (f. 39, fig. 6). Le modèle linéaire est alors disqualifié au profit d'un nouveau mode d'écriture et de lecture :

Le geste littéraire (d'écriture ou de lecture) consiste essentiellement à pouvoir et savoir entendre et faire entendre dans un langage d'autres langages. Il y a toujours au moins une écriture sous l'écriture : le vrai modèle n'est pas « la source », l'influence - ou même la parodie, c'est le palimpseste (cf. Genette, Proust [...] (f. 39-40, fig. 6-7).

La «profondeur» qui est ouverte par l'écriture paragrammatique n'indique pas une source ou origine remise au jour ; l'écriture paragrammatique ne cache pas une « vraie » écriture avec une autre : « on pourrait être tenté de dire : écriture crypto-grammatique $\neq$ écriture phanéro-grammatique - Ce serait faux : le paragramme ne relève pas du à-déchiffrer, il ne comporte pas de secret » (f. 41, fig. 8).

Après avoir mis en valeur la profondeur du texte littéraire, le séminaire s'arrête sur l'expérience du lecteur menacé par une sorte de fuite en avant, comme Saussure dans sa quête effrénée des anagrammes cachées dans les vers latins : "Imaginons la tragédie pour un "scientiste", un savant de l'époque positiviste : ne pouvoir se déprendre de l'improbable : par là même, S[aussure] participe déjà à cette autre logique, à ce double qui fonde l'anagramme »(f. 36, fig. 3). Dans Les Mots sous les mots, en 1971, Jean Starobinski développera ce point, en citant des lettres de Saussure :

Quand un premier anagramme apparaît, il semble que ce soit la lumière. Puis quand on voit qu'on peut en ajouter un deuxième, un troisième, un quatrième, c'est alors que, bien loin qu'on se sente soulagé de tous les doutes, on commence à n'avoir plus même de confiance absolue dans le premier ; parce qu'on arrive à se demander si on ne pourrait pas trouver en définitive tous les mots possibles dans chaque texte ${ }^{13}$.

En butant contre l'infinitude du langage et du texte, Saussure commence à douter de l'intentionalité des anagrammes, qu'il continue néanmoins à traquer derrière tous les vers anciens qu'il étudie. Starobinski, qui lit Saussure dans le contexte des théories de textualité développées en France dans les années soixante et soixante-dix, perçoit, derrière les tentatives frustrées de ce dernier pour assigner de l'autorité à sa lecture, la découverte de l'infinitude de langage : «Est-ce le vertige d'une erreur ? C'est aussi découvrir cette vérité toute simple : que le langage est ressource infinie, et que derrière chaque phrase se dissimule la rumeur multiple dont elle s'est détachée pour s'isoler devant nous dans son individualité14. » Barthes, pour sa part, reconstitue cette interprétation de l'œuvre de Saussure selon l'opposition derridienne entre la voix et l'écriture :

Saussure et les Anagrammes : ambiguïté constitutive : découvre le langage comme espace (fait fondamental aujourd'hui) mais reste prisonnier de la voix, de la phoné (critique de Derrida), alors que l'espace ne peut entraîner qu'à fonder le langage sur l'espacement, càd sur la trace, la différence, càd sur l'écriture (f. 37, fig. 4).

Si, dans la séance précédente du séminaire, le supplément derridien avait été associé à la logique linéaire de la rémunération, la trace et la différence derridiennes sont ici convoquées pour explorer le langage comme espace à multiples dimensions.

\section{Inventer une écriture critique}

Se pose alors la question des modalités pratiques de production de l'écriture paragrammatique, et Barthes fait appel à l'opposition bakhtinienne entre, d'une part, l'écriture «monologique, monophonique, monogrammatique, simple, univoque, linéaire, logique, épique, scientifique, descriptive, théologique, dont l'espace est le 1 » (f. 41, fig. 8) et, d'autre part, l'écriture «dialogique, stéréophonique, polygrammatique (ou paragrammatique), double (ou multiple), bi-voque, tabulaire, ménipéenne, carnavalesque, dont l'espace est le $2 »$ (f. 40, fig. 7). Il propose de classer quelques artistes selon cette opposition : Racine, Valéry, Montherlant, Taine, Quinet, Beethoven, «Johny Haliday » seraient

13. Ferdinand de Saussure, cité dans Jean Starobinski, Les Mots sous les mots, Paris, Gallimard, 1971, p. 132.

14. Ibid., p. 153 
monologiques ; Proust, Klossowski, Bach, Stravinski, Chaplin, Antoine seraient «poly » ou «stéréo » (f. 41). Barthes confesse que cette classification n'est qu'un jeu, mais il ajoute que l'établissement même d'une antithèse relève du style paragrammatique : « la "nuance", énoncé à prétention monologique : l'antithèse est au service d'un sur-intelligible, d'un deux fois intelligible » (f. 41, fig. 8).

Il ébauche ensuite une liste de techniques d'écriture qui relèvent du paragrammatisme (f. 42-43, fig. 9-10), liste où l'on perçoit les germes de bien des réflexions à venir, notamment sur l'amphibologie 15 , mais où se donnent également à lire les bases de sa propre évolution stylistique : comment en effet pourrait-on parler monologiquement d'un objet qui ne l'est pas ?

Cette liste, qu'il qualifie d'ailleurs d'ébauche initiale, fait apparaître combien la notion de paragrammatisme se révélera importante pour son style à venir.

Si l'on s'avisait de dresser une liste des figures d'écriture de Barthes lui-même, chacune des techniques ici relevées y figurerait. Déjà, derrière l'idée d'allégorie et d' « écriture de soi sur écriture de moi » (f. 43, fig. 10), on entend le futur Roland Barthes par Roland Barthes. À cette époque, Barthes cherchait depuis quelque temps un nouveau mode d'écriture critique, capable de relever les défis posés par les découvertes de linguistes et d'autres penseurs contemporains sur l'ambiguïté linguistique. Dans Critique et vérité, publié en 1966, quelques mois avant le début de ce séminaire, Barthes soulignait la nécessité d'une écriture critique qui soit ironique et contesterait la distinction étanche entre critique et écrivain. La mise au jour du texte paragrammatique dans les écrits de Kristeva rend cette tâche plus pressante encore. Barthes avoue pouvoir facilement reconnaître les attributs d'une écriture paragrammatique, mais que la vraie difficulté est de savoir comment en parler :

Il n'y a donc pas de difficulté fondamentale au niveau des procédures de découverte. Mais au niveau des procédures d'exposition? Dans quelle écriture allons-nous décrire le paragrammatisme? mono- ou polygrammatiste. Cela pose la question de la paragrammatique, ou discours concernant l'espace du langage, c'est-à-dire sur la littérature, c'est-à-dire discours critique (f. 43-44, fig. 10-11).
Le Barthes des années soixante-dix devait transformer sa pratique rédactionnelle critique en une écriture/lecture qui fasse place aux désirs et aux besoins subjectifs, mais en 1966-1967, il suit encore la première voie. C'est sans encore renoncer à l'écriture dite critique qu'il commence, à ce moment, à réfléchir sur ses propres pratiques de rédaction et exprime son besoin d'inventer une écriture qui soit en accord avec les nouvelles théories littéraires dont il est de plus en plus question dans ses propres textes et dans ceux de ses contemporains. Or, le problème du discours critique traditionnel réside dans l'explication qu'il cherche à donner d'un texte, explication qui a pour but et fin d'annuler le paragrammatique et de limiter la prolifération du sens. Utiliser un tel discours pour décrire le paragrammatisme, ce serait donc œuvrer contre chacun de ses principes :

Reconnaître - et même louer - le paragrammatisme en usant d'une écriture monologique [...] c'est coiffer libéralement le polygrammatisme par un monogrammatisme qui le « comprendrait ». Ceci est évidemment intolérable. [...] Le problème fondamental aujourd'hui n'est donc pas du tout d'élaborer une théorie critique, une théorie de la littérature, de la production littéraire, c'est de décider si une langue critique est possible et quelle elle doit être (f. 44, fig. 11).

Comment le lecteur-critique peut-il négocier sa position de lecteur-écrivain, et réussir à rendre compte sans le trahir du paragrammatisme du texte qu'il lit ? Barthes considère brièvement la proposition de Julia Kristeva : se servir du langage mathématique pour échapper à la logique de la phrase indo-européenne16. Il conclut cependant qu'un tel discours serait facilement récupéré et disqualifié comme formaliste et scientifique. Une deuxième option lui semble plus féconde :

Inventer une écriture critique qui soit elle-même paragrammatique ? Seule lueur vers laquelle il faut aller. Quelles voies, quels actes, quels modes d'inscription? On ne sait encore ; par ex : voies de truquage subreptice d'un monologisme apparent ; ou encore critique-roman, à forme quasi mons-

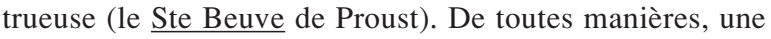
chose sûre marque le départ de cette invention fondamentale :

15. On peut lire le Roland Barthes par Roland Barthes comme la continuation de cette liste des figures paragrammatiques. Voir, par exemple, la section sur « l'amphibologie » (OC, t. IV, p. 650-652).

16. J. Kristeva, « Pour une sémiologie des paragrammes », art. cit., p. 146. 
rejet, pour parler de la litt[érature], de l'écriture des écrivants : il faut écrire : ce qu'on dit de l'écriture doit être écrit ; et tout ce qui s'est parlé ici et se parlera ici - précisons-le nettement pour terminer cette introduction - doit être reçu comme relevant des « procédures de découvertes » et non tes procédures « d'exposition» (f. 45, fig. 12).

La boucle se boucle : cette question de la forme même que doit prendre l'écriture critique, Barthes a entendu Derrida la formuler à Baltimore ; il l'a retrouvée dans les textes de Kristeva qu'il vient de lire. Dans un entretien de 1971, il reviendra d'ailleurs sur l'importance du paragrammatisme pour lui : «Si vraiment j'avais un jour à faire la critique de mon propre travail, je centrerais tout sur le "paragrammatisme"17. » Le terme reviendra peu dans la suite de son œuvre, mais la notion déterminera la pratique barthésienne de l'écriture, avec un fort investissement éthique. De fait, dans Critique et vérité, Barthes avait déjà ébauché un mouvement en ce sens, critiquant ceux qui considèrent « que l'œuvre s'offre au déchiffrement (ce en quoi on la reconnaît symbolique) », mais se contentent de «mener ce déchiffrement au moyen d'une parole elle-même littérale, sans profondeur, sans fuite, chargée d'arrêter la métaphore infinie de l'œuvre pour posséder dans cet arrêt sa "vérité"18 ». L'écriture critique devra donc recourir à cette même écriture paragrammatique qu'elle cherche à décrire, créant ainsi un continuum entre forme et contenu. En cette année 1966-1967, Barthes n'est pas le seul à le penser : on l'a vu, Derrida et Kristeva méditent aussi le besoin d'une nouvelle langue critique qui puisse décrire l'ambiguïté des textes paragrammatiques sans trahir cette ambiguïté en recourant au discours critique assertif. Kristeva formule ainsi la question à la fin de « Pour une sémiologie des paragrammes » :

Le problème se pose alors pour le sémiologue de choisir entre le silence et une formalisation qui a la perspective, en tâchant de se construire elle-même comme paragramme (comme destruction et comme maxime), de devenir de plus en plus isomorphe aux paragrammes poétiques 19 .

Quelques mois plus tôt, dans son intervention au colloque de Johns Hopkins, Derrida avait lancé le même défi : trouver un mode de discours qui ne trahirait son intention contestataire et ne retomberait dans les structures discréditées qu'il cherche à critiquer. Le séminaire de 1966-1967 doit se lire dans ce dialogue ; il marque un tournant définitif dans la pensée de Roland Barthes, tout en indiquant la direction que prendra son écriture à venir.

Écrites et centrées sur la question de l'écriture, les notes que nous donnons ici ne connurent cependant qu'une réalisation orale, puisqu'elles n'aboutirent point à une publication $^{20}$. Mais elles mettent aussi en jeu de façon plus complexe la dialectique de l'écrit et du parlé dans la genèse de la pensée et du discours : elles furent rédigées dans le sillage de l'exposé de Derrida entendu à Baltimore peu avant, et elles poursuivent l'entretien qui s'était ébauché à cette occasion (les actes du colloque en gardent la mémoire); elles sont nourries de la discussion qui a préparé un exposé que doit faire Julia Kristeva dans les semaines à venir. Ces notes ont, par ailleurs, servi de support à deux autres présentations orales : à la State University of New York, tout d'abord, le 28 février 1967, puis lors du cours professé à Genève en 1971-1972. En témoignent divers ajouts marginaux postérieurs, comme l'acronyme « suny » (fig. 2 et 6), ou la mention du livre de Jean Starobinski paru en 1971, Les Mots sous les mots (fig. 2). Plus encore que les parties du séminaire qui ont abouti à une publication, ces notes confirment en outre que l'année 1966-1967 a bien représenté un tournant épistémologique et stylistique majeur dans la carrière de Barthes. Et cela d'autant plus que l'espace oral et amical du séminaire autorise une liberté de réflexion et de propos qu'interdit le texte publié ; il permet par exemple l'aveu direct d'une défaillance de la parole du locuteur lui-même : « [Ceci : a été mon problème dès le début : problème informulable, incommunicable puisqu'il faut précisément disposer d'une langue pour le communiquer] » (f. 45, fig. 12). Mais on voit que ces mots sont notés entre crochets, comme si Barthes les avait écrits pour lui-même ou s'était demandé s'il devait les dire, ou bien même s'il ne devait les dire que comme une parenthèse restée écrite.

17. $O C$, t. III, p. 1019.

18. $O C$, t. II, p. 798.

19. J. Kristeva, « Pour une sémiologie des paragrammes », art. cit., p. 146. 20. Pourtant, Barthes avait continué de travailler sur ces notes. Pour les développer, y insérer de nouvelles idées ou des citations découvertes ou remémorées plus tard, il recourait à des fiches, dont le point d'insertion est indiqué dans la marge des feuillets, par exemple ici figure 6. 
Maria O'Sullivan est professeur de littérature française à l'Université de Cork (Irlande). Elle a soutenu une thèse sur l'écriture critique de Roland Barthes à l'Université d'Oxford en 2010. Ses intérêts portent sur la théorie littéraire et sur la littérature française contemporaine. Ses dernières recherches concernent les questions du temps, du mouvement, de l'immobilité et de l'image dans l'œuvre de Barthes.

maria.osullivan@ucc.ie 


\section{Description matérielle}

Cette cinquième séance du séminaire 1966-1967, consacrée à " L’Espace du langage (le paragrammatisme) » est conservée à la BnF, sous la cote NAF 28630. Le dossier comporte treize feuillets écrits uniquement sur le recto. Ces feuillets sont paginés de la main de Barthes et vont de «34» à « 45 ». Ils sont de format $21 \times 27 \mathrm{~cm}$. Est associée à ce dossier une des célèbres fiches, si caractéristiques de l'écriture de Barthes. Elle est appelée dans le texte par le chiffre romain «VI », figurant dans la marge du feuillet 39.

Le premier de ces feuillets, le 34, est le résultat d'un collage. Le bas du feuillet 34 a été agrafé sur un autre feuillet (papier de type différent, plus épais et filigrané « Marais », tous les autres étant filigranés « Extra Strong Basseau »).

Les instruments d'écriture sont surtout le stylo à encre noire, mais aussi, avec chaque fois une signification précise, le crayon gris, le stylo à encre bleue ou le feutre rouge. L'usage de couleurs différentes témoigne également de temporalités différentes. Ainsi, le passage au crayon gris caractérise clairement des insertions tardives. Si le manuscrit date de janvier 1967, l'inscription marginale au crayon qui figure sur le feuillet 35 sous la forme de «+ les mots sous les mots », titre de l'ouvrage de Jean Starobinski, ne peut de ce fait être antérieure à 1971.

Même chose pour le stylo bleu, sans qu'on puisse savoir exactement à quel moment (postérieur à l'écriture de ce texte) il intervient. À titre d'hypothèse, on propose que le stylo bleu date du moment où Barthes revoit ses notes pour la conférence qu'il donnera le 28 février 1967 à la « State University of New York ». D'où sans doute les deux inscriptions marginales en bleu « Suny » sur les feuillets 35 et 39 (ici suivi de la date " 28 fév ». Pourrait relever de la même campagne de révision le retraçage des grands crochets droits en bleu sur le feuillet 38, qui pourraient signifier l'omission de ce paragraphe à l'oral prévu. Si notre hypothèse est juste, elle vaudra aussi pour l'inscription marginale en bleu sur le premier feuillet du dossier (f. 34) « 1966-67 séminaire sur le Discours de l'Histoire / Introduction », qui serait une sorte de rappel à l'auteur pour lui-même pour qu'il explique à son auditoire de quel ensemble cette conférence fait partie.

Reste à dire un mot sur l'usage du rouge. Dans ce petit dossier, on ne repère qu'une seule occurrence, dans le bas du feuillet 39, où Barthes rappelle à propos de la notion de « linéarité » qu'elle a été « repoussée par Derrida »; ce nom propre est suivi d'abord d'un astérisque noir, puis d'un astérisque rouge, qui est lui-même réitéré et complété dans la marge par le chiffre romain «VI» encerclé de rouge. Ce chiffre romain renvoie à la fiche VI (reproduite ici à la fin des fac-similés, fig. 13) qui sous le thème "linéarité » comporte une citation extraite de La Grammatologie de Derrida ; au milieu supérieur de la fiche on lit en rouge le même chiffre romain, suivi de «p $39 »$, qui indique que cette fiche est à insérer au feuillet 39 du texte du séminaire.

Si cet inédit porte clairement les traces visibles d'un avant-dire (fréquence des abréviations, soulignements, deux points, retraits, tirets et phrases nominales), c'est un écrit extrêmement organisé, ce qui se voit à travers les nombreuses marques du plan. Le chapitre 5 (entouré par un rond) est subdivisé en trois sous-chapitres (chaque fois le chiffre est encadré), respectivement subdivisés en «a) - », «b) - », «c) - », après quoi, marquées encore par des retraits, il y a de nouvelles sous-unités en « $1-»$, « $2-»$, etc. Il suffit de regarder par exemple le feuillet 37 pour comprendre cette organisation structurée de bout en bout de l'espace page. C'est incontestablement une tentative du discours de la maîtrise - qui néanmoins n’a jamais été publiée. 
Nous avons fait : longue introduction sur la linguistique du discours, bilan actuel, surtout prospectif : 1) au delà de phrase, substitution de la Rhétorique, catégories fondamentales de la grammaire, le discours rémunération de la langue.

Pour terminer En tout ceci, langage imaginé selon une vue classique (et provisoirement nécessaire) comme une ligne, un mono-plan, ou encore une temporalité simple (consécutive). Nous allons, pour terminer, fappeler donner au langage, au discours, sa deuxième dimension, sa « profondeur » et rappeler qu'il est un espace (et non une ligne).

Nous y serons aidés par Saussure lui-même, les recherches de Bakhtin sur le « dialoguisme » et les prolongements que donne actuellement à ces recherches Julia Kristeva, qui m'a communiqué des textes inédits, dont je ferai état ici - ce dont je la remercie.

1 Les Anagrammes de Saussure

a) - Saussure, homme énigmatique, ou tout au moins problématique. Benveniste : un certain agraphisme de S parce qu' 1 ne pensait ne pouvoir parler du langage que s'il en remettait en cause tous les concepts en même temps. v. Benveniste : un côté Léonard de Vinci. Homme Ce n' est pas un savant simple ; le développement de la linguistique fera apparaître une complexité : une certaine histoire de Saussure sera sans doute possible.

- Le Cours de Saussure : sa gloire unique. Or en fait : i1) de son vivant, grande réputation d'indo-européaniste, nullement de linguiste 2) fait, paraît-il, avec déplaisir et pour de l'argent. Actuellement, soumis à trois vastes critiques : les 3 «fautes » de Saussure : 1) le taxinomisme (Chomsky) 2) le phonologisme 3) la linéarité. (Derrida)

b) - Or, S : une autre œuvre, écrite ou destinée à l'être, mais non publiée : œuvre énigmatique et qui pose bien des questions inattendues : les Anagrammes :

- 99 cahiers, dossiers de tableaux s/ grandes feuilles. Mnss écartés de la publication par la famille, mais dont on entrevoit la parution : cahiers d'exemples d'anagrammes, moisson considérable.

- Actuellement : 1) description des dossiers. Godel. CFS $17(1960)^{1}$

\footnotetext{
${ }^{1}$ Robert Godel, «Inventaire des manuscrits de F. de Saussure remis à la bibliothèque publique et universitaire de Genève », Cahiers Ferdinand de Saussure, ${ }^{\circ}$ 17, 1960, p. 5-11.
} 


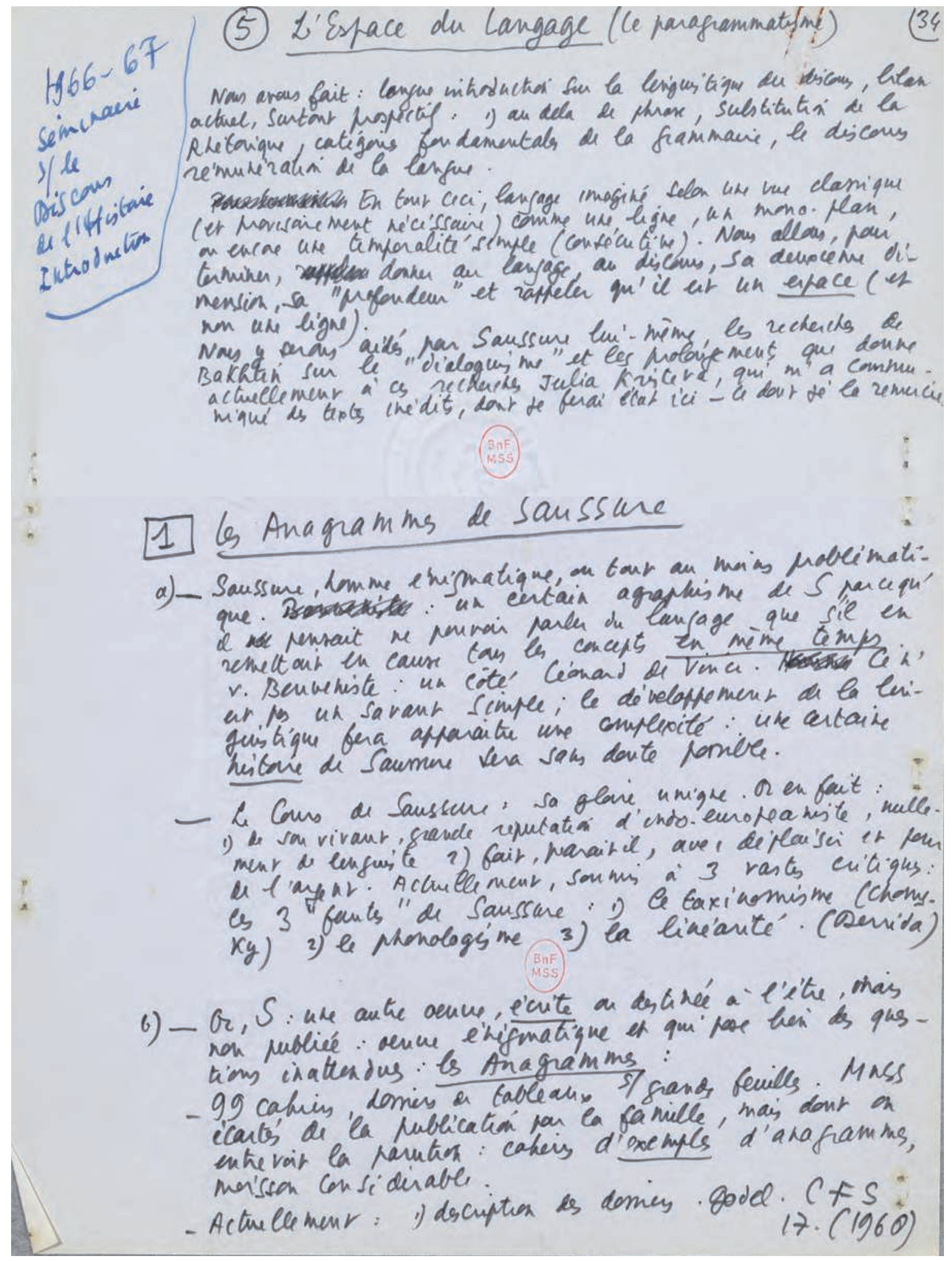

Fig. 1 
2) Fragments présentés par Starobinsky dans Mercure, Fév $64^{2}$.

+ les mots sous les mots

3) 2 lettres importantes de S à Meillet, 23 Sept 1907 et 8 Janv 1908 (C.F.S. 21, 1964) ${ }^{3}$

- qu'est-ce que le paragramme saussurien (ou anagramme, ou hypogramme)?

- paragramme : (Larousse) : faute d'orthographe, emploi d'une

lettre pour une autre - Paragrammatisme : allitération.

- Départ : étude des allitérations dans le vers saturnien latin (vers archaïque, septenaire iambique catalectique (càd dont le dernier pied est incomplet, raccourci) : Chants d' Étrurie, vers dialogués, nés des fêtes paysannes.

Or : $\mathrm{S}$ constate (ou croit constater : ce sera tout le pro-

lettre blème) : «L'allitération c'est-à-dire la corrélation des phonèmes

109 placés à la tête des mots est une partie tout à fait insignifiante d'un phénomène autrement vaste et important ».

Autrement dit : l'allitération est partout. 2 étapes :

a) le diphonisme : éléments phoniques disséminés par couples, en écho : tout vers saturnien : suite d'échos binaires

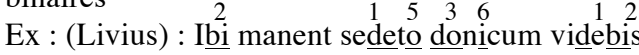

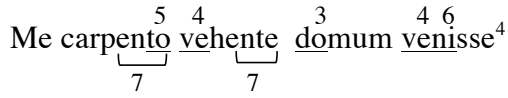

$\underline{\text { Suny }^{5}}=$ cette audition double du vers $=$ «grouillement phonique »

b) l'anagramme (ou hypogramme) : Seconde découverte, ou seconde « audition »: « Les polyphones (les diphones : le grouille-

lettre ment) reproduisent visiblement dès que l'occasion en est

111 donnée, des syllables d'un mot ou d'un nom important pour le texte et deviennent alors des polyphones anagrammatiques $\gg \rightarrow$ On a donc : un nom inscrit dans le vers sous une forme éparse, séparée, espacée.

Taurasia Cisauna Samnīo cepĭt

$$
=\text { Scipio } \quad \overline{\mathrm{a}}+\mathrm{p} \breve{\mathrm{i}}+\breve{\mathrm{l}}^{6}
$$

Ce nom : mot-thème ou mannequin (parfois réduit à anaphoniel ses voyelles : Cornelius : o.e.i.u). Sorte de bruissement figure bruissante plus ou moins formée. L'anagramme se déroule, soit sur un nom qui figure (ailleurs) dans

lettre le texte) soit sur un nom qui n'est pas prononcé, mais 111 qui vient à l'esprit par le contexte

\footnotetext{
${ }^{2}$ Jean Starobinski, «Les anagrammes de Ferdinand de Saussure : textes inédits », Mercure de France, n $^{\circ} 350$, février 1964 p. 243-262. Jean Starobinski devait publier chez Gallimard en 1971 Les Mots sous les mots : les anagrammes de Ferdinand de Saussure; la notation marginale a été ajoutée à l'occasion de la reprise de ces notes pour un exposé fait à Genève en 1971-1972.

${ }^{3}$ En 1964, Benveniste a publié les «Lettres de Ferdinand de Saussure à Antoine Meillet », dans les Cahiers Ferdinand de Saussure, $\mathrm{n}^{\circ} 21$, p. 93-130). Les références marginales à «Lettre» dans les feuillets qui suivent renvoient à ce texte.

${ }^{4}$ Livius Andronicus, L'Odyssée, VI, 295-296 : «Assieds-toi ici et pleure, jusqu'à ce que tu me voies rentrer à la maison sur mon char. »

${ }^{5}$ State University of New York, où Barthes a utilisé ces notes pour une présentation orale, le 28 février 1967.

${ }^{6}$ «Il a capturé Taurasie, Cisauna et Samnium». Cette inscription se trouve sur le tombe de Lucius Cornelius Scipio Barbatus, maintenant dans le musée du Vatican. L'exemple, donné par Saussure, se trouve dans le texte de Jean Starobinski, Les Mots sous les mots, op. cit., p. 34.
} 
2). Fagmens pricente' par Scarobinsky dales (35 Mercure, fer 64.

3) 2 Lettes impatants de S à Neillet, 23 sept

$+\frac{\text { les not }}{\text { tes mot }}$ 1907 et \& Janv 1908 (C.F.S.21, 1964)

- gu'ur agm le paragramme saussavien (on añgramme, on hypogramme)?

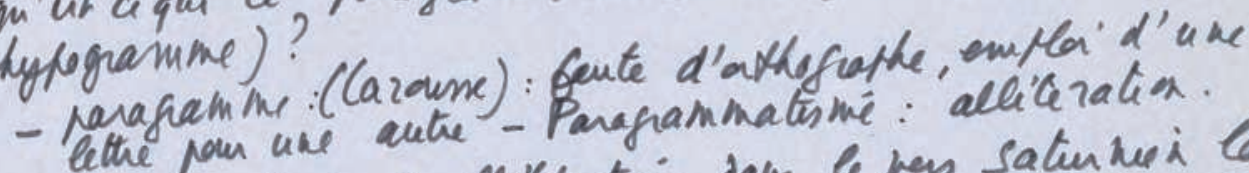
lettre pour une aute - Paragrammatesme: allilirater.

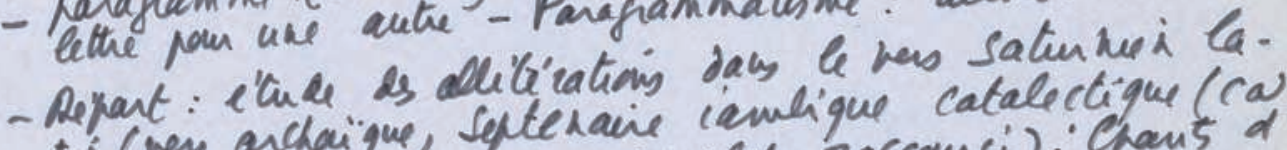
tik (ves orchaigue, setteraire iembique catalectique ( $c a$, Drar a dermin Nid uL incayplet, zaccourci).

Etrunie, tow dialgas', hes ds Gits raysanns. or: S coustate con chir constater conclatia des ponims

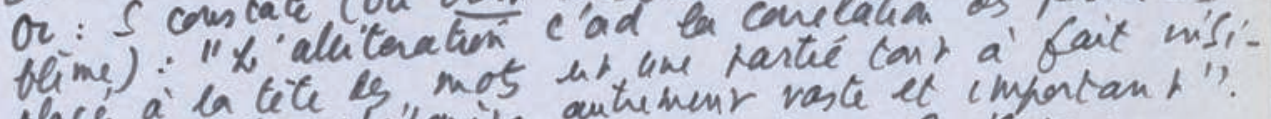

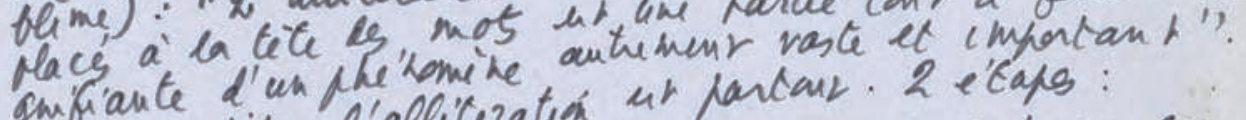
gnifiante d'un phe dit: l'alliciation ur parcaur. 2 e'caps:

Autreneur dit: l'allitents phomigus disse'mins' par con-

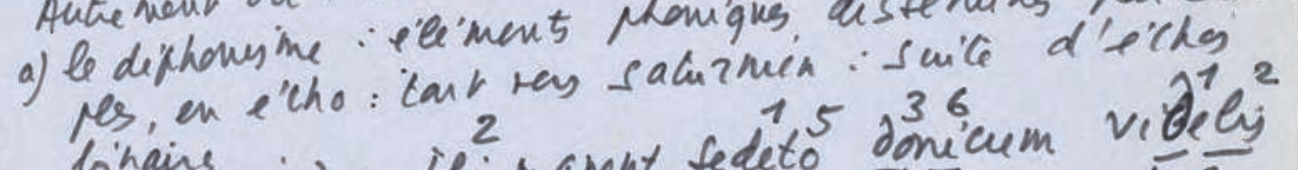

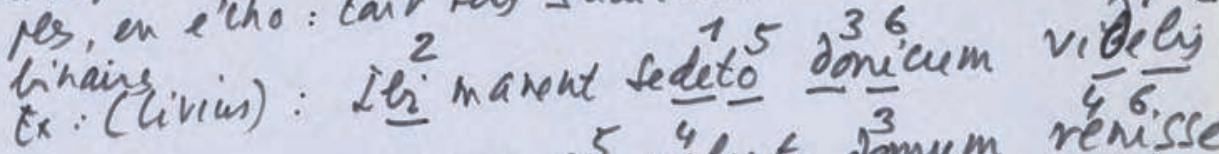
Me carpento velente domum rénisse ulace
109

Ulte

Cltbe III b) l'ana framme (on hyperamme): Seconde diconacite, on EcGade "audition". "Les polythons (les dixhous: le grouille. ment), repoduisenr vifideemerr dis que l'occasen en as donkéc, as tyelabs d'un mot on d'un hom imparauc pom l' texe et devenneur alas as polyptons anagrammatiqus" $\rightarrow$ On a derci un nom insent

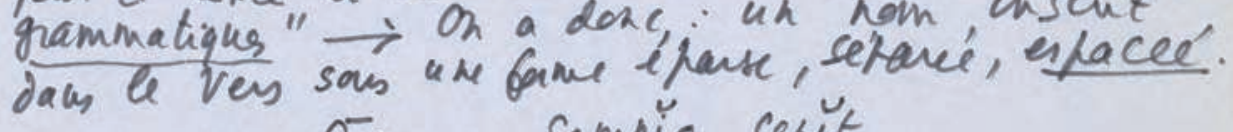

Tamasia Gisanna Samnio cefit

$$
=\text { Sexio } a^{-}+\mu^{\nu}+i_{0}
$$

Ge nom: not-thime ar manrequin (rarfais rident ì

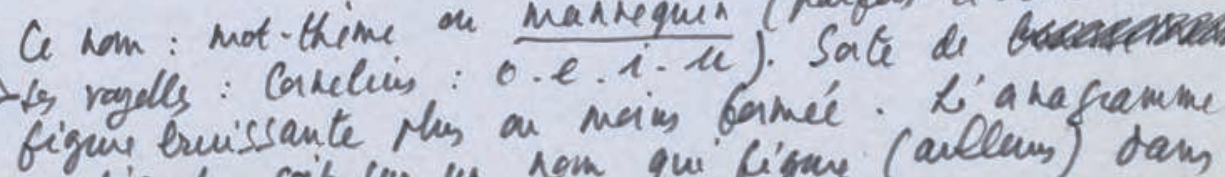

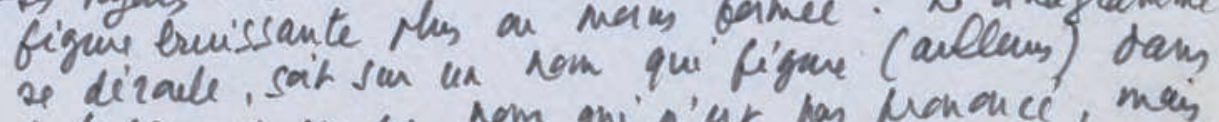

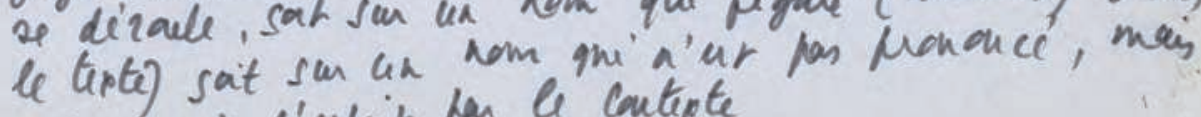
qui veur ì $l^{\prime}$ upuir tas le coutepte

Fig. 2

143 
origine de la pratique anagrammatique : «idée supersti-

lettre tieuse qui a pu suggérer que pour une prière ait son effet,

114 il fallait que les syllables mêmes du nom divin y fussent indissolublement mêlées : on rivait pour ainsi dire le dieu au texte ou on créait un lien que la divinité n' était pour ainsi dire plus libre de repousser ».

une fois

c) - Ici se noue un drame : le phénomène anagrammatique perçu, S. l'entend partout. Véritable voix obsessionnelle, hallucination tyrannique : chez les latins, d' (Ennius à Virgile, Lucrèce, Catulle,

Ovide Tibulle) chez les Grecs (Homère), dans les Vedas germaniques

Stace et même dans toute la poésie latine, même celle qu'on faisait encore vers 1815-1820. La structure, sinon anagrammatique, du moins anaphonique, envahit tout, même la prose : un bruissement signifiant, un double inéluctable.

Drame de « savant » : voit partout ce dont personne n'a jamais parlé (aucun auteur ancien : ou parce qu'allant de soi, ou parce que secret, initiatique) : S. était-il fou, halluciné ? $\rightarrow$ non publication des Anagrammes et cependant y a passé toute sa vie : c'était son œuvre - non le Cours.

D'ailleurs censure de la société scientifique. Imaginons la tragédie pour un «scientiste », un savant de l'époque positiviste : ne pouvoir se déprendre de l'improbable : par làmême, S. participe déjà à cette autre logique, à ce double qui fonde l'anagramme. Nos positivistes n'ont pas de ces vertiges, de ces doubles voix : ils n'entendent rien, ils palpent, comme Thomas. Mais Saussure : conflit entre la Voix et la Science.

d) - La portée des Anagrammes :

- Une vue anthropologique sur la Poésie : Vates : manieur de phonèmes, celui qui sait les distinguer (par ex : il décompose d'abord le mot thème, puis construit ses vers autour des morceaux). Les jeux de la versification sont lettre partis de l'anagramme. Conception qui va plus loin 114 que celle de Jakobson : J. aplatit le paradigme (le diphone) en syntagme ${ }^{7} ; \mathrm{S}$. restitue et maintient une « profondeur » du langage :

$$
\left|\begin{array}{c}
\mathrm{A}^{\bullet} \\
\mathrm{A}^{\prime} \cdot
\end{array}\right| \mathrm{A} \rightarrow \mathrm{A}^{\prime}
$$

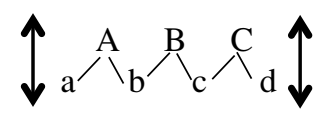

L'espace du langage reste en qque sorte tangible

\footnotetext{
${ }^{7}$ «La fonction poétique projette le principe d'équivalence de l'axe de la sélection sur l'axe de la combinaison» (R. Jakobson, Essais de linguistique générale, op. cit., p. 220).
} 
Geltue

cuigin \& la patique anagrammatiqu "idei fapersa: 136 114

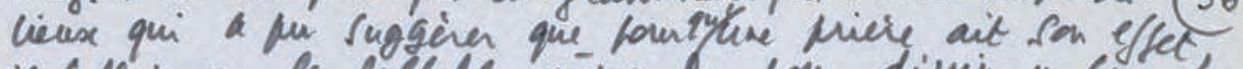
il Gallait que les syllabls nèms on hom divin y funch

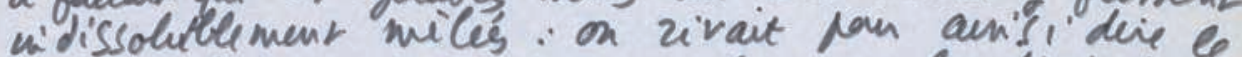

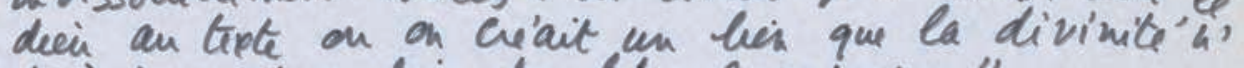
e'tait nom ainsi dire plus lite de repoussa."

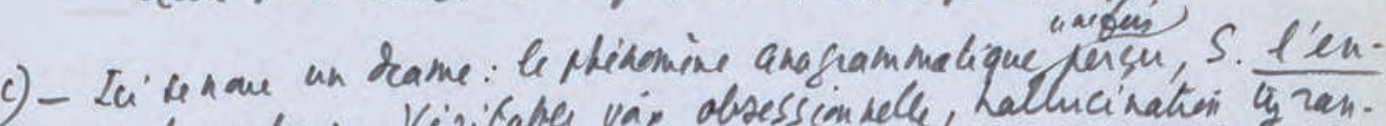
tend partour. Viritaber vaip obsessionsele, hallucination ty ran.

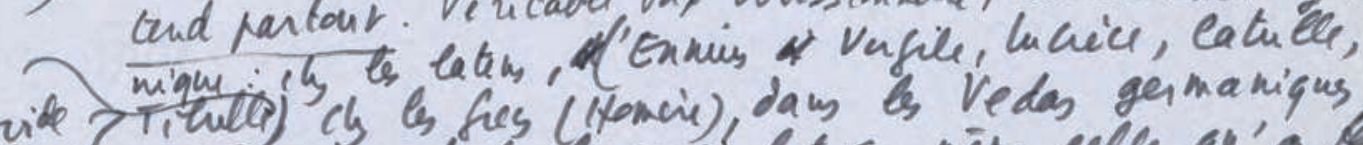

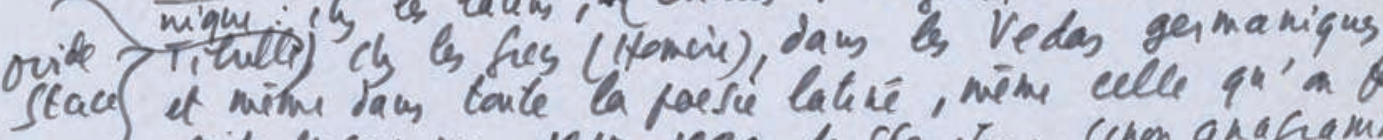

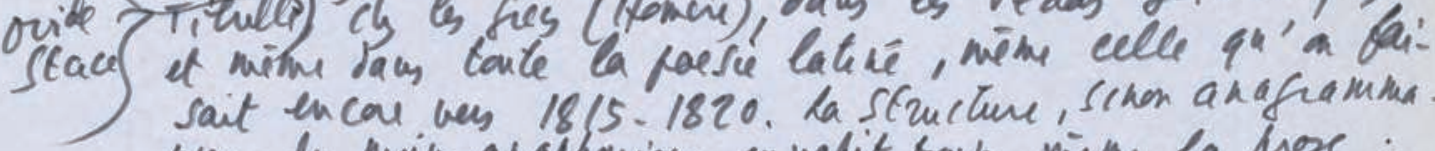

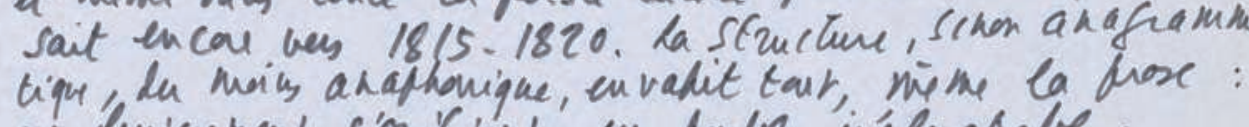
uh herisenent signifiant, un louber inéluccable. arame de "sa vant" " vait partour. 4 denr personve " a jamai, parlí (ancuen autem ancien: on paragiallans de sai, on parceque renet, sniliatique): S. etair it ber, halle. cine'? $\rightarrow$ non publication ds Anaframms et echendanr y a hase' toute sa bie. c'itair son coure - non le cong.

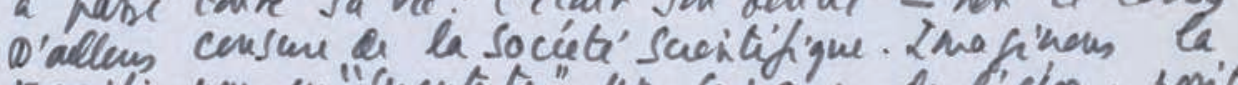

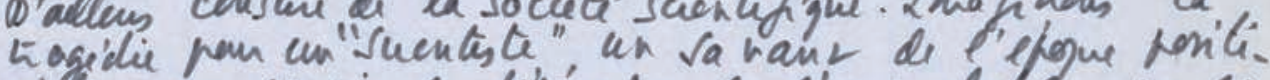
viste: he pouroin a difiendre de l'improbable: Ner la:

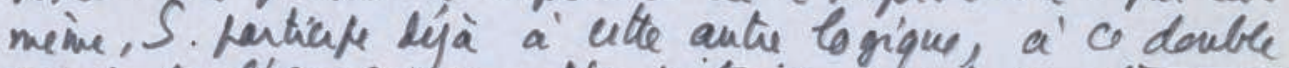

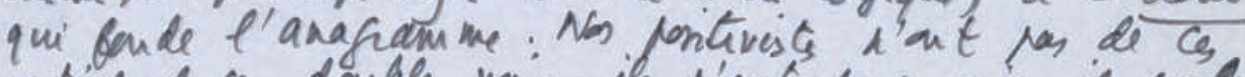

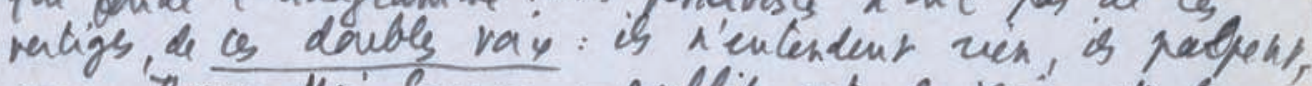
crmme Themas. Mais Saumere: couflit ente la Voris it la Science.

d) - La patei los Anagramms

- Une vare anthopolonighe sur la Poesie: Vates: matucier de

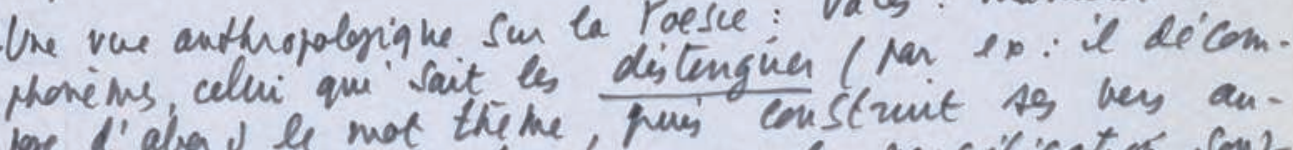
pre d'abad le mot the he, pres conslinit ts ves antoun des maceaup). jeup de la rersification $\operatorname{sen} 2$ lethe pute's d'anagramme. Conception qui va res lain pue celle de Jakolson: J. aplatir le paradigme (le dí phone) en syntagme; $S$. restitue it maniterit une "probs. dem II) du lenjage:

$$
\text { (A':) } A \rightarrow A^{\prime} \text { ( }
$$

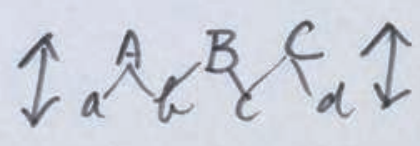

Linpace th langage wite en gque sate tangible 
- Le discours poétique a pour fondement le double:

un vers = la seconde façon d'être d'un nom, d'un mot (d'où : hypogramme : souligner au moyen de fards les traits du visage) donner au mot une façon d'être factice.

- Contestation de la linéarité du langage (Saussure contre le Cours) : autre tempo que celui de la consécutivité simple. Tempo polyphonique, càd en fait extra-temporel : anagramme : «moyenne des impressions acoustiques hors de l'ordre du temps $»^{8}$. En fait, décisif, postulation d'un espace du langage (et non d'une ligne)

d) - Saussure et les Anagrammes : ambiguïté constitutive : découvre le langage comme espace (fait fondamental aujourd'hui) mais reste prisonnier de la voix, de la phoné (critique de Derrida) $^{9}$, alors que l'espace ne peut entraîner qu'à fonder le langage sur l'espacement, càd sur la trace, la différence, càd $\mathrm{s} / \mathrm{l}$ 'écriture.

Ambigüté Oscillation entre 2 métaphores saussuriennes :

lettre 110

- le grouillement (phonique) = l'indistinct, la trace brouillée, l'anti-trace.

lettre 113

- la tapisserie (« la poésie védique est littéralement tapissée d'anagrammes »), càd l'inscription, l'espacement des fils, l'image écrite dans le tapis ${ }^{10}$.

Ceci serait à rattacher au mythe de la Voix (abordée d'une façon métaphysique par Derrida) : la phoné a été le démon de Saussure : il a entendu dans tout vers (dans tout langage) un fond, une figure, une voix (mythique, préhistorique), mais hésitant - explicitement - entre grammé et phoné, il n'a pas su que cette hésitation serait un jour (aujourd'hui) décisive ; et pourtant comme démon, la voix saussurienne comportait à la fois vérité et destruction.

\section{Bakhtin et Julia Kristeva.}

Travaux de B. sur Dostoievsky et Rabelais. Articles de J. K. s/ Bakhtin, Jehan de Saintré et Lautréamont ${ }^{11}$.

a) Bakhtin. Recherches sur le mot romanesque : le mot n'est pas un point fixe de sens pris dans une chaîne linéaire, monovalente, mais une profondeur, un « carrefour », un dialogue : autrement dit, il y a un statut du mot romanesque (et non

${ }^{8}$ J. Starobinski, Les Mots sous les mots, op. cit., p. 47.

${ }^{9}$ J. Derrida avait déjà énoncé sa critique du phonocentrisme dans les deux articles «De la grammatologie [I et II] », (Critique, $\mathrm{n}^{\circ} 223$, décembre 1965, p. 1016-1042 et $\mathrm{n}^{\circ} 224$, janvier 1966, p. 23-53).

${ }^{10}$ Souvenir de la nouvelle de Henry James, The Figure in the Carpet (1896), où un personnage s'acharne, tout comme Saussure, à découvrir un thème secret qu'un auteur célèbre aurait mis dans tous ses livres.

${ }^{11}$ Les articles en question sont «Bakhtine, le mot, le dialogue et le roman » (Critique, ${ }^{\circ}$ 239, avril 1967) ; «Le texte clos » (Langages, $\mathrm{n}^{\circ} 12$, décembre 1968, p. 103-125) et «Pour une sémiologie des paragrammes » (Tel Quel, $\mathrm{n}^{\circ} 29$, printemps 1967, p. 53-75). Les trois textes ont été repris dans Sêmeiôtiké. Recherches pour une sémanalyse (Paris, Éditions du Seuil, 1969, rééd. Points, 1978). Les livres de Bakhtine dont traite l'article de Kristeva sont La Poétique de Dostö̈evski et L'Euvre de François Rabelais et la culture populaire au Moyen Âge et sous la Renaissance. Ces ouvrages ne paraîtront en français qu'en 1970. 


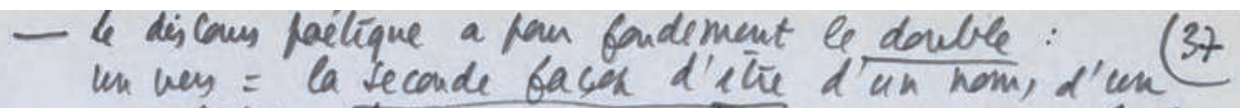
mot (d'ai : Tyrogramme. Sactignes an mayen de far os les tais the visage) dounar an mot une facen d'ithe bactice.

- Contestation de la linéarité de langage (Saumere coutre

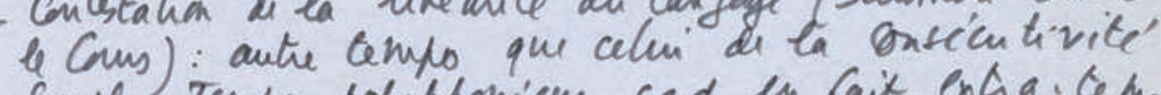
simple. Tempo polyphonique, cad en fait exha. Gem-

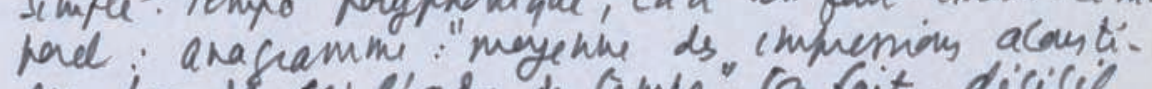
qus has de l'abe du cemps. Ga fait, dicisy potulation d'un upace du largage (et non do lene lifie)

d) - Sausruer ef es Anapramms: amlignite constitutive:

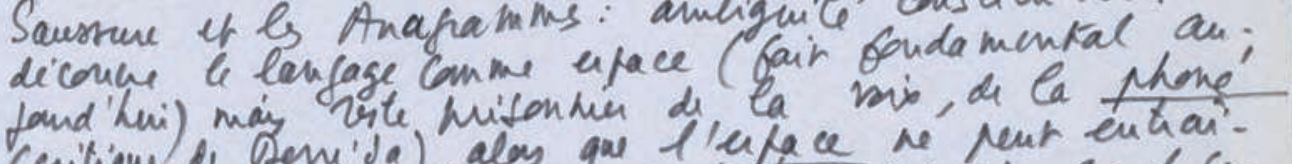

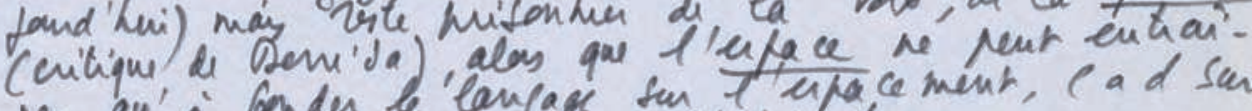

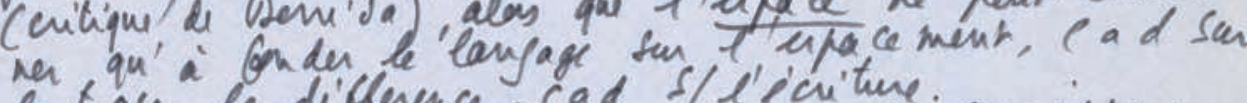
la tace, la difference cad s/l'écriture.

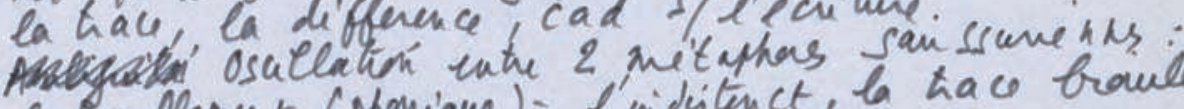

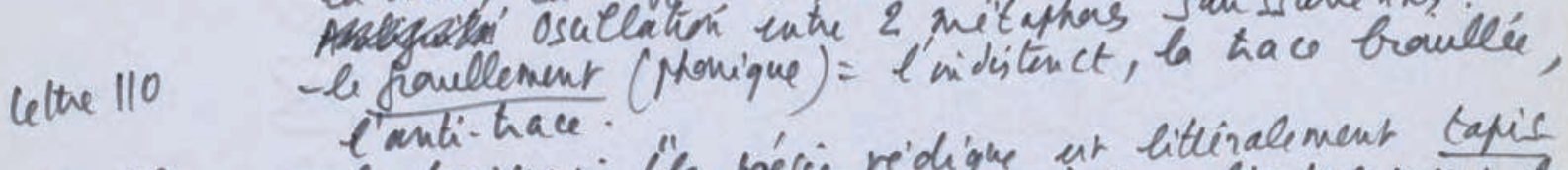

Lethe 113

- la tapisserie "la póscie rédique ur littiralement tapis

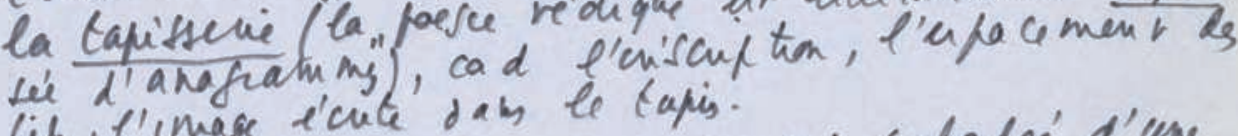
fib, l'image e'crite dats le tapis.

Cei serait a' rattacher an mylhe de la varo (abadee' d'une bace mitaphynigue ter Denida): la Mhone a éle' b demon de Saussure: il a encendu dam tant ves (dases ton $r$ langage) un fand, whe feigure une vas (my chique mikic conque), mais he'sicant - explicitiment - ente gramme et More, il, i'a pas sa que athe hisitation serair en Jom. (mymishic) dicisire, et poutant comme dimon, la vap saussurichne compartait i' la fois ren'te' et descruction

2 Bakthrin et Julia Kisteva.

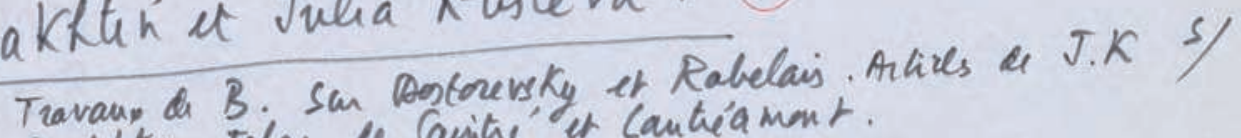
Bakthe, Jehen de Sainte' "er Cautéa mont.

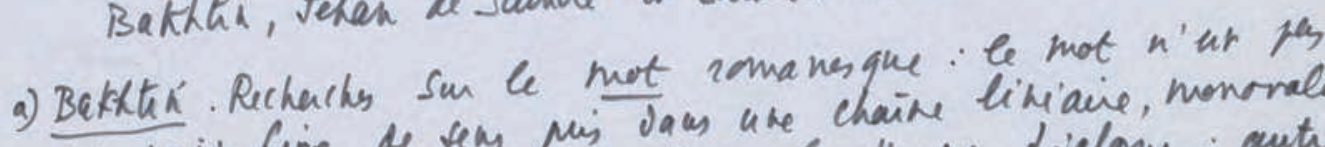
un parit fipe de sems mis Jams are chaine liniaire, monoralen$t e$, mais une pofondem, un "canefan", un Jialgre: autre. ment dit, it $y$ a un scatect an mot zomanerque (es non

Fig. 4

147 
seulement un sens, une définition). Dialogue avec quoi ? non avec le lecteur (vue banale et d'ailleurs insoutenable, connotation morale et vaine du mot dialogue), mais dialogue en chaque mot avec d'autres mots, et les mots des autres, dialogue de chaque écriture avec d'autres écritures, venues du contexte ou actuel ou historique (d'où présence de l'histoire dans l' écriture). Ce « dialoguisme », lorsqu'il est complet, développé, définit un type d'écritures : écritures du Carnaval ou de la Ménippée (Satyre Ménippée : pamphlet collectif de la fin du XVI ${ }^{\mathrm{e}} \mathrm{s}$, du nom du philosophe cynique du $3^{\mathrm{e}} \mathrm{s}$, Menippe, auteur de parodies) ${ }^{12}$.

de la En face : écritures non-dialogiques, « monologiques », linéaires, monologique $\sqrt{ }$ alentes : celles de l'épique, par exemple. Opposition entre Dostoïevs-

et de ki et Tolstoi - Nous reviendrons sur cette grande dualité des écritures ${ }^{13}$.

(Exposé le 20 janvier)

b) Julia Kristeva : contributions suivantes :

1 - Dégage bien la substitution - pour le texte littéraire - du modèle tabulaire au modèle linéaire (cf les Paragrammes de Saussure)

(et littéraire)

2 - Montre que le code poétique n'est pas un sous-code du code total (version « morale » de la hiérarchie des langages), mais qu'il est la seule infinité réelle du code [[chaque phrase (chaque séquence) peut être attachée à la précédente comme un élément qui ne lui appartient pas : le texte est peut être une chaîne d'ensembles vides qui se retourne sur elle-même $\rightarrow$ il est donc impossible d'établir une

Paragr. contradiction dans l'espace du langage poétique : «Dans le langage poétique, la contradiction n'est pas non sens mais définition, la négation y détermine et les ensembles vides y

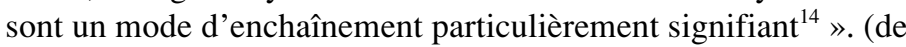
zéros signifiants)]]. Ainsi le texte poétique (paragrammatique) conteste non seulement le système du code humaniste avec lequel il dialogue (ceci très net chez Lautréamont), mais sa propre texture. [Ceci va plus loin, est plus radicale que l'ambiguïté

jakobsonienne).

3 - Formule en termes modernes le « dialoguisme » bakhtinien : le texte est à la fois écriture et lecture : «dans le paragramme d'un texte fonctionnent tous les textes de l'espace lu par l'écrivain ${ }^{15} »$. [illis.] Le texte étranger : absorbé soit comme réminiscence, soit comme citation. Le dialogue n'est donc pas entre le sujet et le destinataire, l'écrivain et le lecteur, mais dans l'acte même de l'écriture [illis.] : l'interlocuteur est un texte, le sujet est aussi un texte. Non pas intersubjectivité, mais inter-textualité : le texte étend la division et la différence des écritures.

\footnotetext{
${ }^{12}$ Voir J. Kristeva, « Bakhtine, le mot, le dialogue et le roman », Sêmeiôtiké, op. cit., p. 103-107.

${ }^{13}$ Ibid., p. 90-91.

${ }^{14}$ J. Kristeva, «Pour une sémiologie des paragrammes », Sêmeiôtiké, op. cit., p. 129.

${ }^{15}$ Ibid., p. 120.
} 


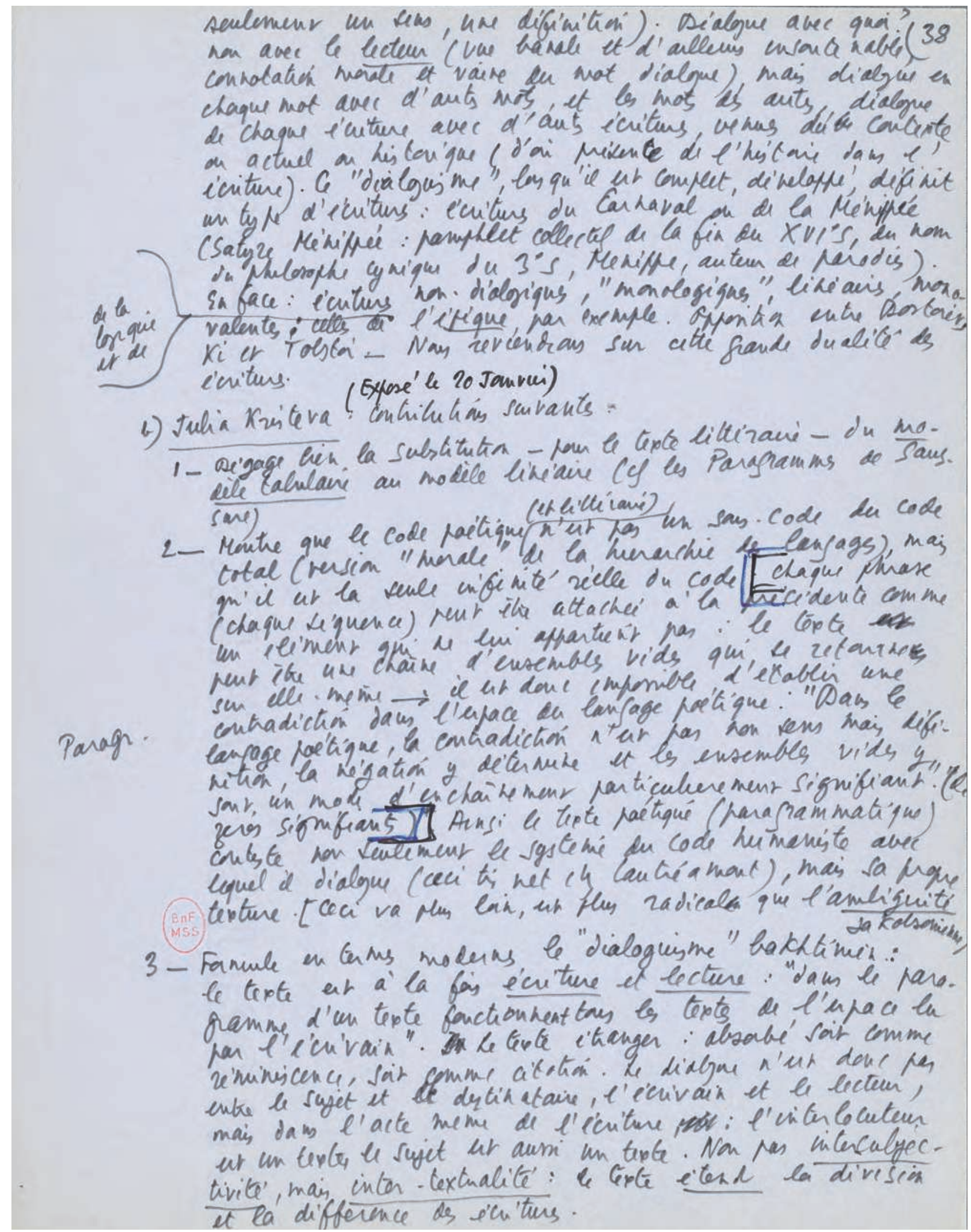

Fig. 5 
4 - Précise la portée de l'écriture paragrammatique :

Contestation du monologisme, destruction de l'écriture mono-

logique, levée du tabou de l'interdit attesté par toute monovalence : à l'écriture du 1 (interdit, sens, véri-

morale té, science, logique) s'oppose l'écriture du 2, qui la détruit, mais se détruit aussi elle-même. L'écriture paragrammatique manifeste le caractère historique des oppositions censure/liberté, conscient/inconscient, culture/nature Dans l'écriture paragramm, le 1 est implicite, quoique (cette écriture ne veut pas « rien dire $\left.{ }^{16} »\right)$ mais transgressé ; s'attaque à la loi du 1 (Dieu, Morale, Société, Science), condamnée, selon le mot de Lautréamont, pour son manque de modestie : la modestie de l'écrivain : ne pas s'égaler au 1, à Dieu, refuser de codifier.

\section{(3) Monographisme et Polygraphisme : perspectives}

Nous retrouverons Julia Kristeva en conclusion à propos du problème : comment parler du paragrammatisme. Mais ici, dès maintenant, qques réflexions sur le langage double, et les deux écritures : la monographique et la polygraphique, la monologique, et la dialogique.

\section{Reprise du principe paragrammatique.}

- Une écriture qui dialogue avec une autre écriture (Carnaval, Ménipée). Un exemple : Sade : écriture essentiellement paragrammatique : l'écriture « érotique » entre sans cesse en dialogue avec des écritures littéraires, philosophiques, lyriques, aphoristiques, mythologiques : contagion métonymique qui détruit à la fois le « beau style » et l'érotique.

Par ex : dans : «Un instant moment, un moment, Mesdemoiselles, dit Delbène en cherchant à rétablir l'ordre... » ou : «L'extrême vénération qu'on avait pour les ordres de la supérieure fit mettre à leur exécution la ponctualité la plus entière » : paragramme : admonestation libertine et apostrophe professorale, le texte érotique est écrit sous et avec le texte scolaire, jésuite ${ }^{17}$.

- Il existe donc bien un espace tabulaire du langage litt La linéarité : affirmée par Saussure ds son Cours, démentie par lui dans ses Anagrammes, rectifiée par Jakobson (l'accord en musique $)^{18}$, repoussée par Derrida $* * 19$ : Cette linéarité apparaît

VI bien comme radicalement détruite. Le geste littéraire (d' écriture ou de lecture) consiste essentiellement à pouvoir et savoir entendre et faire entendre dans un langage d'autres

\footnotetext{
${ }^{16}$ Souvenir de la lettre de Rimbaud à Georges Izambard (13 mai 1871) : « Ça ne veut pas rien dire ».

${ }^{17}$ Voir Sade, Fourier, Loyola (1971), Euvres complètes, op. cit., t. III, p. 699-868.

${ }^{18}$ R. Jakobson, Essais de linguistique générale, op. cit., p. 165. Derrida note cette question de la linéarité temporelle et l'accord en musique dans son texte « De la grammatologie II », op. cit., p. 39.

${ }^{19}$ Fiche VI, voir fig. 13.
} 
4- Précise la patei de l'éciture paraframmatique.

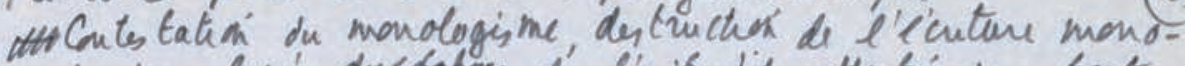
logique, levé dultabias d' l'cicudit atter té par tarte

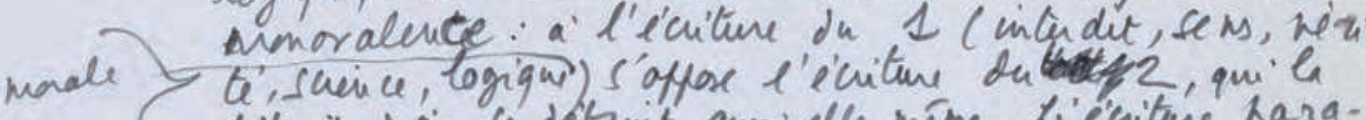
ditruit, mais a détruit ausi elle. méme. b'éciture paragrammatignie manifeste le caractere hitaique ds appoitics consere / beberti', conscuent / enconsuent, culture / hature Dam l'eviture pragramm, le 1 ut impliciti,

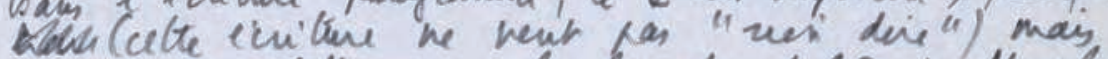
tramsione'; s'attaque à la bi du 1, (Bui), Monale,

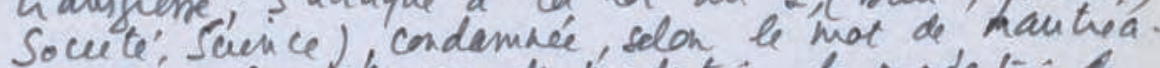

sut

(3) Monographisme et Polygraphesme parpectivs

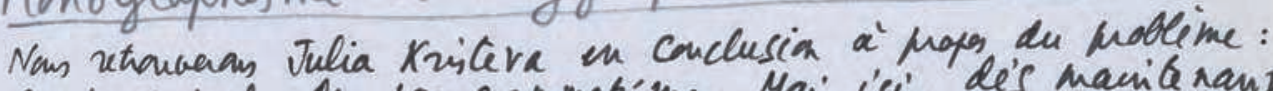
comment porles on paragrammabime. Mais ici's dis macia ranz qqus riflexion sur le langage dabe, et les deas e'criturs: la monographique et la palygraphique, la monolojigue, ex la

1. Repuse du Mincipe paraframmatique.

- Ure e'viture qui'dialgue avec une autie icriture (Carrave,

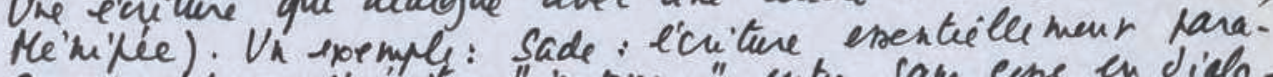
sanmatique. l'ecuiture "elotique" in be sam cerse en dialo.

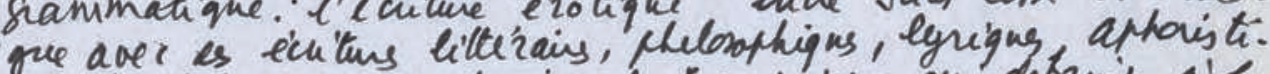
qus, Mythologiqus: contagia metongmique qu detruin $a^{\prime}$ la fis le "beau style" et l'érotaque.

$\mathrm{Par}$ er: da cs "Va wisbor moment, un homent, Mesdema. oeles, dit Delbène in Chuchant a' re'tablir l'a dre...", on.

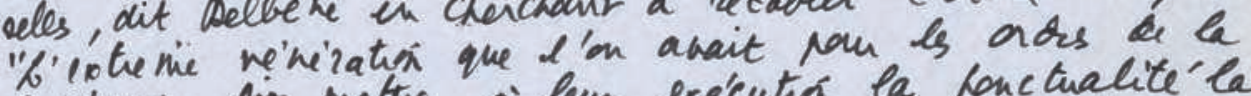
sufinerive fit nethe a' leur rxe'cutin la ponctualite'la Mes enterie" : taraframme. admonetation libertiri is

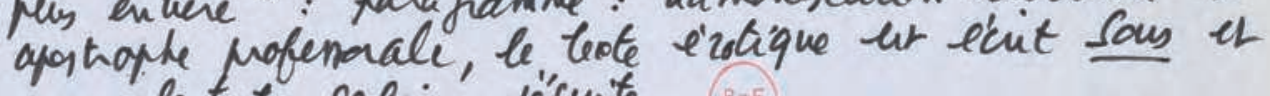
avec le tapte scolaire, de'suite.

- Il exste onc lis un expace talularie de lanjage litt. La lire'arite's affir mée par Saussere \& San Guy, de'mentié tar eni Jam ses Anaframms, rectifié par Jakolson (l'accard en

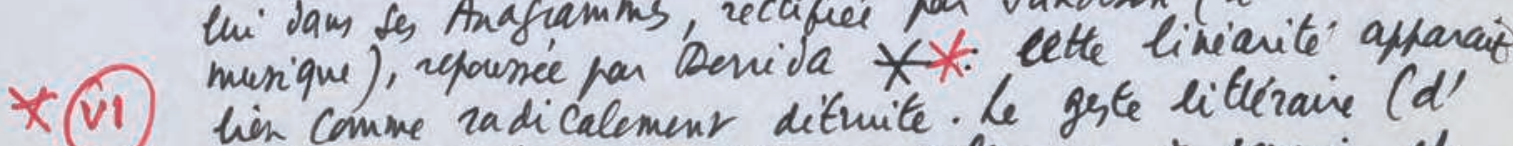
lien comme radicalemenr ditruite. Le geste litiliane (d') ícriture on de lecture ensiste essentiellement à pourai et cavoir entendre it facie entendre dass an lengags d'ants 
langages - Il y a toujours au moins une écriture sous (40

l'écriture : le vrai modèle n'est pas « la source », l'influen-

ce - ou même la parodie, c'est le palimpseste (cf.

Genette, Proust. palimpseste et la surimpression des objets de

l'espace et du temps, la transverberation ${ }^{20}$ ) - On pourrait

dire aussi : le mot (le texte) est scénique : comporte un

proscenium, une avant scène, un plateau et un lointain ;

mais ceci ne doit pas être hiérarchisé en soi (seulement dans

certains cas par jeu) : le «fond »n'est pas plus important (com-

me d'ailleurs au théâtre) : les écritures ne sont pas à « déchif-

frer » selon une apophantique ${ }^{21}$; elles sont des «moyennes acous-

l'anagogie ${ }^{22}$ tiques ou graphiques », hors du temps, de la consécutivité ou

est une illu- de l'étagement - on pourrait dire : l'écriture est

sion stéréophonique, parler de stéréographie (stéreo : solide), de stéréogramme.

- Précisons bien que le dialogue qui constitue l'écriture est intérieur à l'écriture : ce n'est pas un dialogue entre des « objets » mis bout à bout, ce n'est pas une stychomythie : un dialogue simultané (cf. eertains sur un autre plan, certains textes de Butor). L'autre texte n'est pas au bout, hors du premier. Le dialogue se fait toujours avec une autre écriture, non avec une personne ; moyennant cette précision, si l'on prend des personnes, les objets, et les entités, comme des écritures, comme des inscriptions, l'éventail stéréographique est très large : écriture de la'auteurpersonne, de l'auteur-auteur (institutionnel), du narrateur, du destinataire, du personnage, du contexte culturel (antérieur ou contemporain), du contexte historique.

\section{Les deux écritures}

Toute écriture est stéréophonique : stéréophonie, fait de langue, indissolublement lié au langage, qui est toujours inscription historique. Seule écriture non stéréophonique : la mathématique (en fait elle est hors de l'opposition mono/stéréo). Mais la nature stéréophonique de l'écriture est diversement affirmée et diversement assumée. Or comme cette diversité elle-même est codée, càd comme l'écriture dispose de certains signes de stéréophonie ou de monophonie, on est en droit de parler d'une grande opposition archétypique entre 2 écritures (même s'il y a des cas particuliers incertains)

A - une écriture dialogique, stéréophonique, polygrammatique (ou paragrammatique), double (ou multiple), bi-voque, tabulaire, ménipéenne, carnavalesque, dont l'espace

est le 2 .

\footnotetext{
${ }^{20}$ Gérard Genette, « Proust palimpseste », Figures I, Paris, Éditions du Seuil, 1966, p. 39-67.

${ }^{21}$ Dans la pensée d'Aristote, est apophantique une assertion qui peut objectivement être jugée vraie ou fausse selon sa formulation même.

${ }^{22}$ Littré : «Anagogie. Terme de théologie. Ravissement de l'âme dans la contemplation des choses divines, ou efforts qu'elle fait pour découvrir le sens mystique de quelque passage de l'Écriture. »
} 
langags - Il y a tayair an main une e'criture -5 (4) l'eniture - He hai morele n'ur ras "la Sance", l'uiqetuen. ce - ar mine la parodie" c'ur le palimpseste (of. genette, Prourt palemyseste et la serimpionia l'clace er su Gms, la tausrerberatiox) - O pourrait due aussi: le mot' (le tete) ur scemique: cupate un prosceniuem, une avant siene, an Matian et un lasitain. mai, ceci ne dait Nas itre hieraichisecs en sa' (senlemenr day

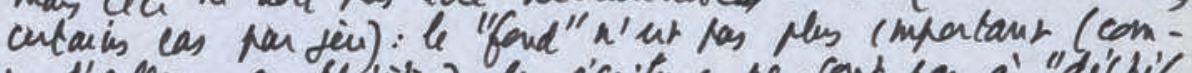

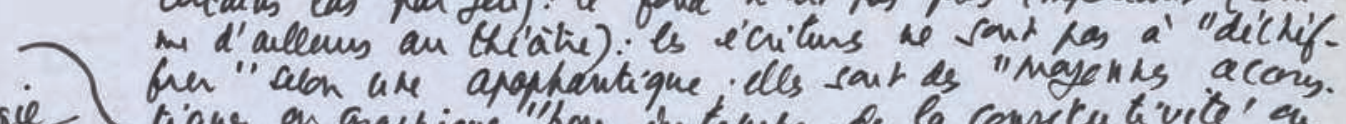
e'anagggie tiqus, a graphiqus,'

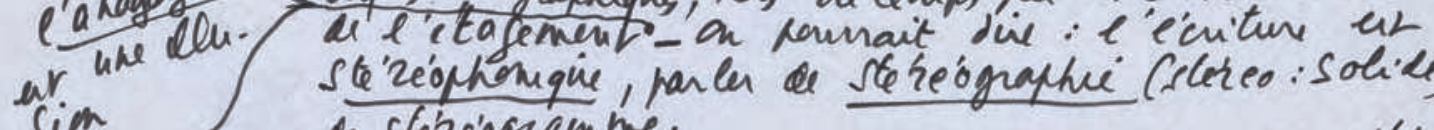

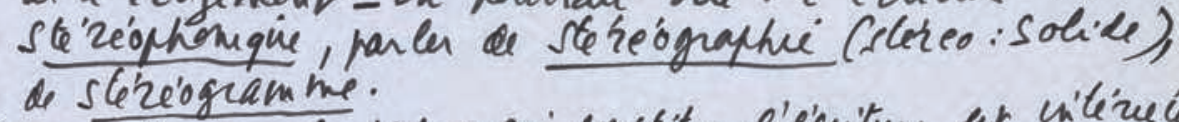
dezéogramme.

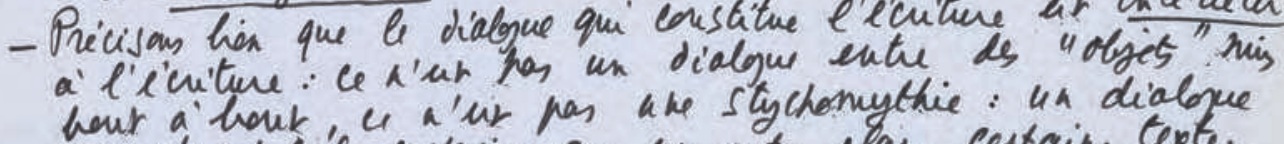
schivetare' ( ' ${ }^{\prime}$.

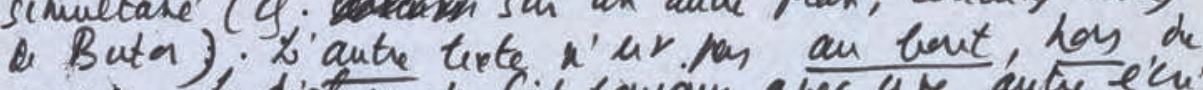

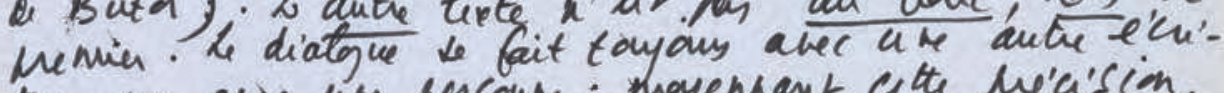
twe, non avee une rersonre; mogennant cethe re'a'sion, si l'on prend les personis, cls objes, et ls entites,

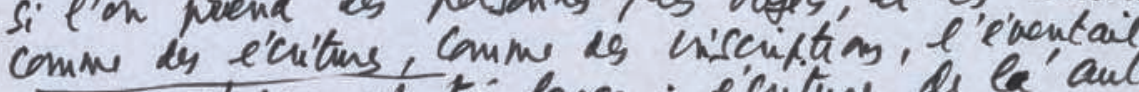
stereographique it ty' lange : e'cuturs de la' autrun. hersone, de l'autem - auteur (cistilutionnel), du rarra. tem, du'dstinatarie du prvanage, du contepte cultu. vel (anternein an contemprain), du con (iple hitaique.

2. Les denp e'cuiturs.

Trute e'cuiture ur stiriophonigne: Stireophomie, fait de lonfue, undissobubeiment lie' an langage, qui us bay jous criscripten

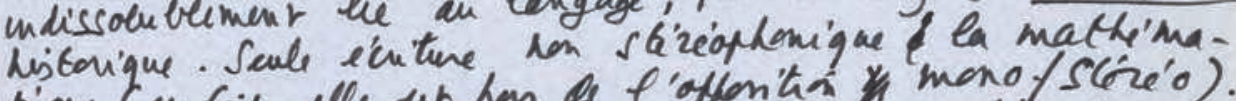
b'que (en (ait ille ur has de l'offoritan mono. (Scréo).

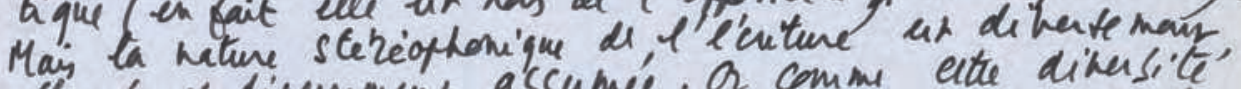
affermée ut direasemenr assumce. O comm ethe dires site elle. mime ar codée, cad comme l'écriture dispose de certains signs de slézéaphomie an de monophonie, on er en boit de parter d'une pande apposition arche'ty rique enter 2 e'miturs (mème s'il y a ds cas particulius incer. taim)

A - ure écriture vialoyique, stiréaphomigu, polignammatique (m paragrammatinne), double (a multiple) ti-raque,

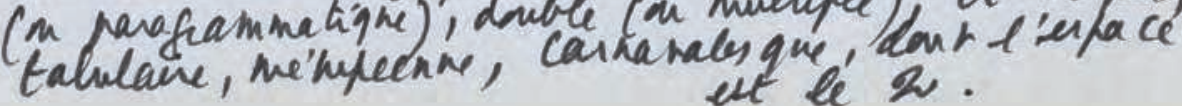

Fig. 7

153 
B - une écriture monologique, monophonique, monogrammatique, simple, univoque, linéaire, logique, épique, scientifique, descriptive, théologique, dont l'espace est le 1 (l'indivis)

Nota : on pourrait être tenté de dire : écriture crypto-grammatique $\neq$ écriture phanéro-grammatique - Ce serait faux : le paragramme ne relève pas du à-déchiffrer, il ne comporte pas de secret. Paradoxalement, c'est peut être le l'écriture monographique qui peut l'offrir à une certaine complexité de lecture - non par rapport à l'écriture elle-même (qui est « claire ») mais par rapport à la personne référente : il y a des écritures monologiques qui s'offrent très bien à une critique thématique. L'écriture monologique peut être signée-, et cette signature peut être secrète (c'est le sens littéral de monogramme) : ne pas confondre signature et écriture.

- Donc 2 grands territoires, 2 pôles graphiques, celui de la Science, de la Théologie, et celui de la Voix unique, et celui de la Ménipée, de la Voix multiple. Auteurs se répartissent à peu près immédiatement et infailliblement. Citons en vrac :

a) du côté des Mono : Racine, Valéry, Montherlant, Taine, Quinet, Beethoven, la musique sérielle, Jean Gabin, les acteurs du Français...

b) du côté des Stéréo, ou Poly : Corneille, Sade, Michelet, Lautréamont, Joyce, Proust, Klossowski, Bach, Stravinski, Charlie Chaplin ${ }^{23}$

Jean Gabin est mono, Jouvet poly. Braque, mono $\neq$ Picasso, Poly - Johny Haliday est monologiste, Antoine est poly dialogique etc

[Bien sûr, il s'agit d'un jeu ; mais il fait partie de l'exposé paragrammatique qui de s'énoncer à travers un classement qui préfère l'antithèse à la «nuance »: la «nuance », énoncé à prétention monologique : l'antithèse est au service d'un sur-intelligible, d'un deux fois intelligible.

- Sur ces deux écritures, sur l'opposition, 3 remarques marginales (càd concernant les marges de l'opposition) :

a) Malgré la tentation, ne pas confondre polygraphisme, ménipéisme et Rire. La duplicité des écritures, même la parodie n'est pas du tout liée au comique, ni réciproquement. Julia Kristeva (commentant Lautréamont) ${ }^{24}$ indique bien que en tant que

\footnotetext{
${ }^{23}$ Sur Charlie Chaplin, voir Barthes, «Le pauvre et le prolétaire », Mythologies (1957), CEuvres complètes, op. cit., t. I, p. 700-702.

${ }^{24}$ "Le rire comme censure est réfuté au même titre que la censure du rationalisme : l'ironie ("rire comme un coq") et Voltaire ("l'avortement du grand Voltaire") sont des ennemis du même ordre » (J. Kristeva, « Pour une sémiologie des paragrammes », art. cit., p. 132).
} 
B- une e'criture monologique, monopharique, nono.

(4) grammatique, siniple, uhivoque, linearie, logique, e'riqu, suintifique, descrittur, the'dógigue, eons $l^{\prime}$ apace ur el 1 (l'u'divis)

Nota: an pannair ite tente' de ine. e'criture cysto-sammatique f

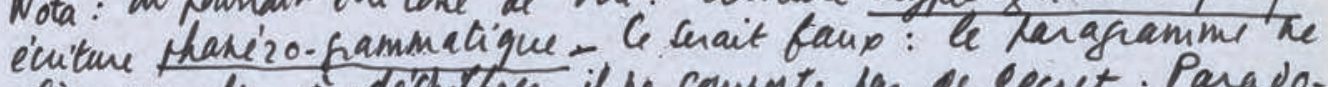
relive pas du a-dechifferer il re comprate pas a secret. Para doxalement, c'ur peur itu ledx l'écuiture monographique qui

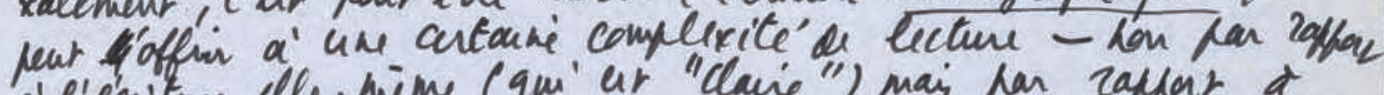
a'l'ecriture elle. mime (qui' ar "clarie") mai par rappar a la pessonse reférente: id $y$ a de e'citurs monolgigus qui's ofe-

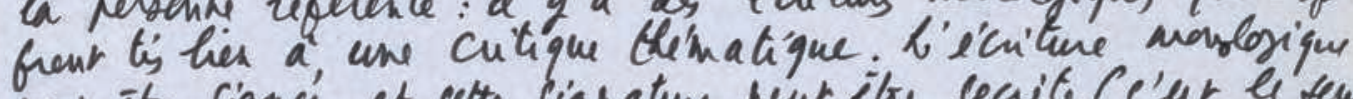
pur, itas signce, et cette sighature peur itue secile (e'ar le sels eittial a monogremme): he pas confon he signative et éciture.

- Done 2 sanes territais, 2 pots graphiqus, celen de la Sacince, a la Thiologie openis a la vaip un'que, et cellui de ba

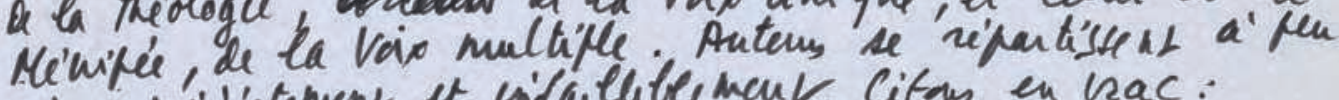
is immidiatemens et cifálliblemenv. Citas en rac:

a) In cote' ds Mono: Racine, Valizy Montherlant, Tarie,

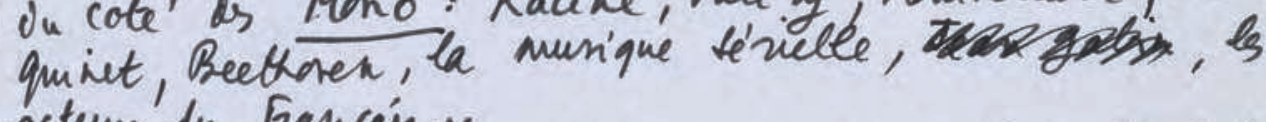
acteuns du Farcás...

b) In cite' des SGirés, an Poly.: Carille, Sade, Michelet, coutiamanr, Jayce, Prant, klossorski, Bach, Straviski,

Jean Galix at mono, Jounet poly. Braque, mono $\neq$ Picasso,

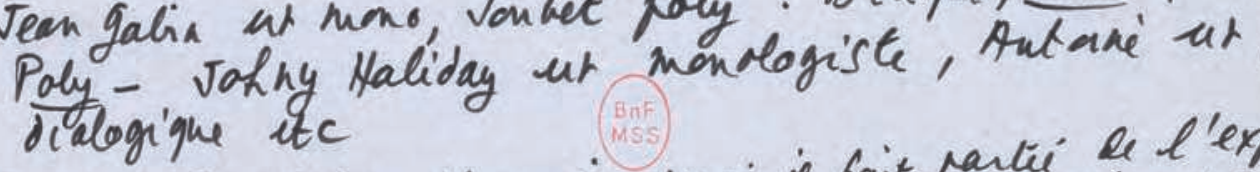

IBuis sin, il J'ajit d'un jea, mai a fait rartie Le l'expose' pragrammatighe de 'f'nonas a'carey un classenent qui prefore $l$ entickese a' la "huance". la "nuance", énoncé a' petarta' monologique . l'ankichese ur an sercice d'an sur-licilligible, d'un diurforis vilillifible.

- Sur ces 2 ícriturs, sm l'appon'tín, 3 umarqus marginals (cad concerant les narge a l'ofpon'tin'):

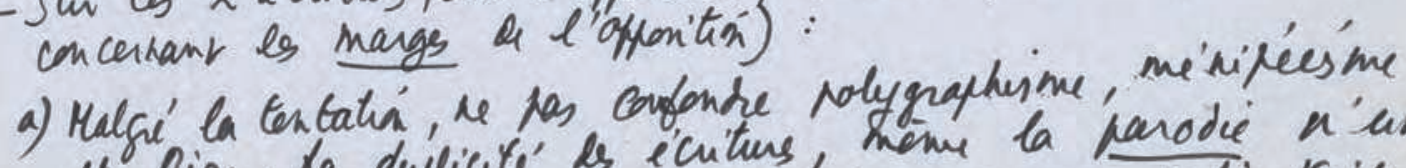
$u$ Rire. Ia duplicite' ls écriturs, mene la parodie n'ut

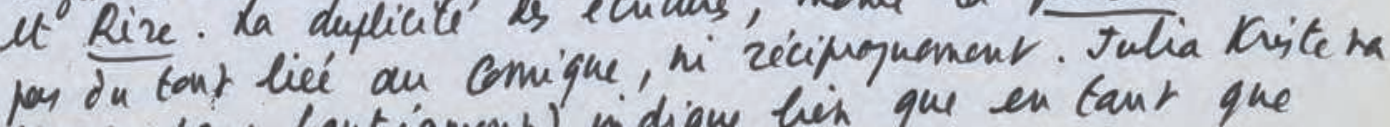
(commentanr Lautiamonr) indique his que en taur que

Fig. 8

155 
censure, le Rire est en somme du côté du 1, de (42

l'interdit, du monographisme (chose curieuse, Saussure avait déjà noté que l'anagramme n'était certes pas inconnu des Comiques, mais que la fréquence y était bien lettre 118 moins grande); le pamphlet est en général monologique. Molière et Ionesco le sont, car ils recourent à une logique du 1 (même à l'envers). Le rire ne peut accepter accéder au polygraphique que s'il est incertain, s'il est destructeur non de contenus, mais de sa propre forme : or en général faire rire implique une prééminence terroriste du sujet : le rire est théologique ${ }^{25}$.

b) Même tentation à éviter pour les écritures automatiques : elles ne dialoguent pas. Sans vouloir assimiler le surréalisme à l'écriture automatique, le surréalisme paraît au dép proche des régions du discours monologique.

c) Peut-on imaginer une écriture transcendante à l'opposition, càd hors-système ? Nous poserons la question à la fin, à propos de la langue critique. Disons que ce ne pourrait être qu'une écriture qui éliminerait à la fois la parodie et la théologie. On peut risquer que Mallarmé a été très proche de cette écriture, ceci d'une façon encore très énigmatique.

\section{Modes, actes, gestes de l'inscription paragrammatique.}

Quels sont les actes, les «figures » par lesquels on peut accomplir, dans une écriture, l'espace paragrammatique ? Comment produire l'écriture polygraphique ? Quelles sont les opérations modernes d'inscription, analogues à l'anagramme dans la par un théorie sausurie

1. Toutes les formes d'écritures parodiques, lé si légèrement, fragmentairement que ce soit (parodie même au seul niveau d'un mot), et dont le modèle n'est pas absolument repérable). Toutefois la parodie, la «citation »doit être sans guillemets ; le guillemet, instrument scientiste, est monologique. Polygrammatisme : oblitère la distinction entre langage-objet et méta-langage. (de même pour les réminiscences) (Redisons encore : ce qui fait rire est toujours entre guillemets).

2. Toutes les formes d'équivoques. Ex : longue équivoque obscène

Machiavel dans le Chant des Vendeurs de pignons, de Machiavel

96 (précisément dans les Chants du Carnaval ${ }^{26}$ )

\footnotetext{
${ }^{25}$ Dans $S / Z$ (1970), l'ironie et la parodie sont qualifiées de monologiques et donc de «classiques » (« Menée au nom d'un sujet qui met son imaginaire dans la distance qu'il feint de prendre vis-à-vis du langage des autres, et se constitue par là d'autant plus sûrement sujet du discours, la parodie, qui est en quelque sorte l'ironie au travail, est toujours une parole classique » (Euvres complètes, op. cit., t. III, p. 155). Qui plus est, le rire a en soi un aspect fortement « terroriste », puisque castrateur (voir p. 158 et p. 283).

${ }^{26}$ «Chant des vendeurs de pommes de pin » dans la section «Chants de carnaval », Euvres complètes de N. Machiavelli, Paris, Garnier, 1867, p. 121.
} 


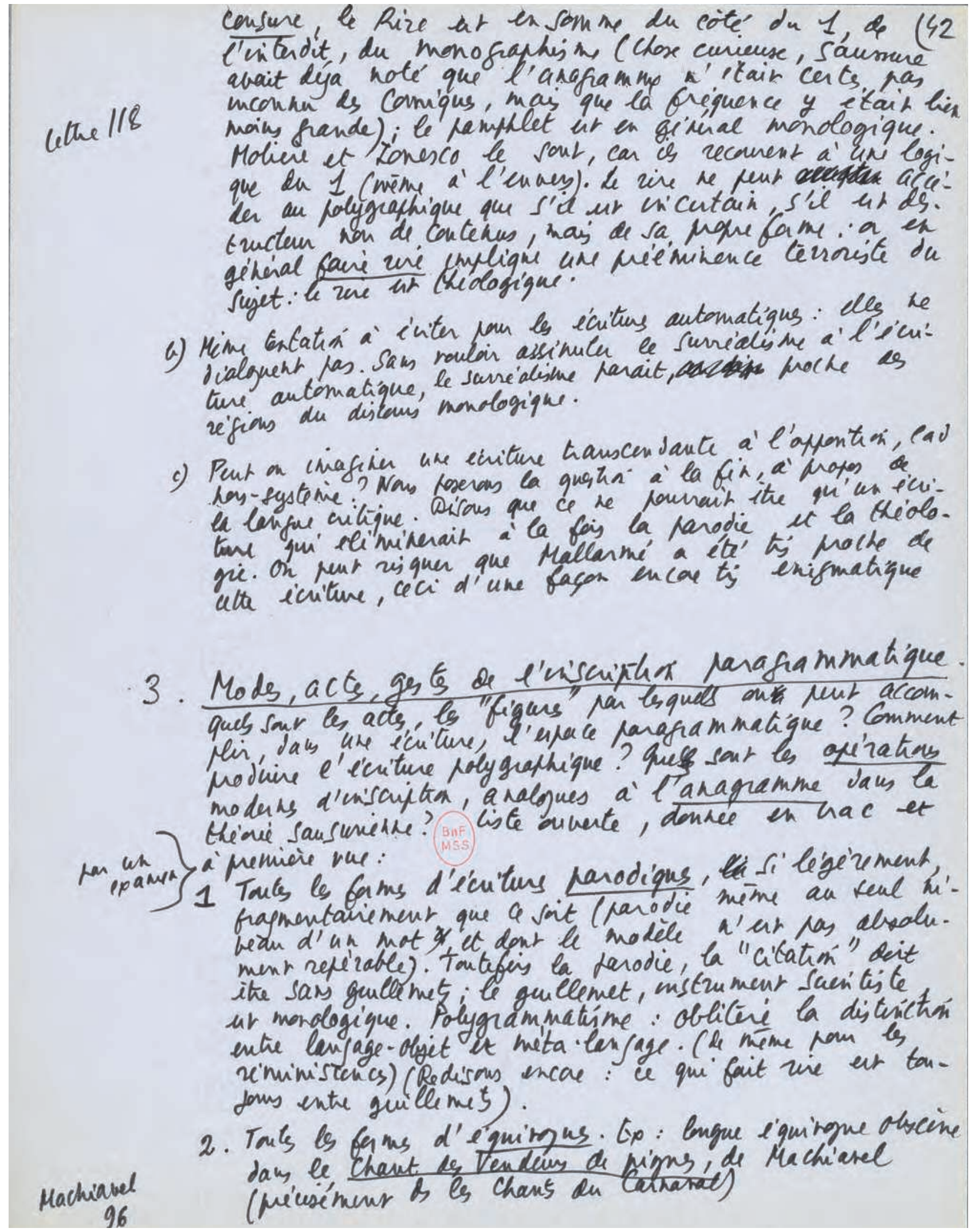

Fig. 9 
3 - Les insertions, enjambements, enrobements visant à dédoubler l'histoire et à conjuguer plusieurs écritures narratives (entorses à la linéarité et à l'ordre naturel et au syllogisme).

4 - Les galimatias, jargons et pédanteries, pourvu que leur parodie soit incertaine - incertitude qui est signalée à qui sait lire par le contexte général de l'œuvre - et plus par

précisément la déception du $\mathrm{S}$ finale du Sé, càd par l'athéologisme du texte - ou encore par les attaques des monologistes.

5 - Les allégories, et notamment les allégories intellectuelles, conformément à cette remarque de Blanchot : «Les idées sont rarement

s/ Cyrano satisfaites des exposés précis qu'on fait d'elles. Elles aspirent passionnément à la fiction, elles ont besoin de rentrer dans le monde $^{27} \gg$ : toute rentrée dans le monde de l'idée, toute inscription du monde sur l'idée.

6 - Les contre-métaphores, càd les métaphores prises et développées à la lettre : métaphores qui se réalisent. Kafka et la Métamorphose (Marthe Robert) ${ }^{28}$; Cyrano et Blanchot : mourir de chagrin (« condamner à une mort qui n'est faite que du chagrin de mourir $»)^{29}$; recueillir son dernier soupir; je t'aime tant que je te mangerais. «Métaphores qui se réalisent par de vraies métamorphoses $\gg(\text { Blanchot })^{30}$

7 - La copie (l'imitation), le double pupitre de Bouvard et Pécuchet. On peut mettre ici la parodie de soi même, écriture de soi sur écriture de moi (nous en verrons des cas en histoire $)^{31}$, le dialogue destructeur du je sacralisé.

8 - Les anacoluthes : changements de logiques ou de sens intervenant subrepticement entre le début et

Gramm. la fin d'une même phrase. Ex : Derrida : «La rationalité »- mais il faudrait peut être abandonner ce mot pour la raison qui va apparaître à la fin de cette phrase $^{32} \ldots$

9 - D’une manière générale toutes les connotations (idée que je suis heureux de reprendre mais seul à défendre) pourvu qu'elles soient artificielles, déclarées, données à lire comme telles, non-naturelles, bref inscrites.

Cette liste - qui n'est qu'un début - montre qu'il est possible de concevoir et même de programmer une vaste description de l'écriture paragrammatique. Mais ici se pose une question Il n'y a donc

\footnotetext{
${ }^{27}$ Maurice Blanchot, «L'homme noir du XVII ${ }^{e}$ siècle », Saisons, n 2, 1946, p. 69-79, repris dans M. Blanchot, La Condition critique. Articles 1945-1998, Paris, Gallimard, coll. « Les Cahiers de la NRF», 2010, p. 56.

${ }^{28}$ Marthe Robert, Kafka, Paris, Gallimard, 1960. Voir «La réponse de Kafka » (1960), dans CEuvres complètes, op. cit., t. II, p. 398.

${ }^{29}$ M. Blanchot, op. cit., p. 58.

${ }^{30}$ Ibid., p. 57-58.

${ }^{31}$ Barthes fait ici référence à un texte qui reviendra dans la deuxième partie de son séminaire : la deuxième préface que Michelet a écrite, en 1869, pour son Histoire de France. Voir Michelet, Histoire de France, Lausanne, Rencontre, 1965, t. I, p. 53 : «En rentrant, d'un élan aveugle et généreux, j'écrivis un mot vif pour ce mourant qu'on pillait pendant l'agonie. Ces lignes juvéniles, étourdies si l'on veut, mais sans doute excusables comme mouvement du cœur [...] me font rire encore. »

${ }^{32}$ J. Derrida, « De la grammatologie I », op. cit., p. 1023.
} 
3- tes vifertion enjambenens eurobements risant (43 $a^{\prime}$ didoubler I'histair et a' bonjuger reuneuis ecu' turs narratios. (entars a' la line'arite' a' l'ade haturel

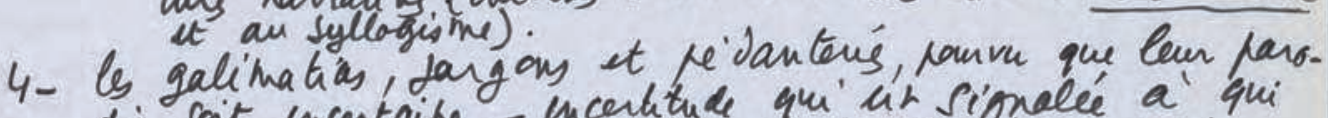
die sait uncertaite - encertitude qui'u's sionolée a' qui sait live rau le ontapte géréral a l'aenare, et Mhes

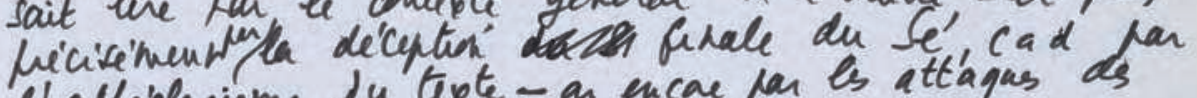

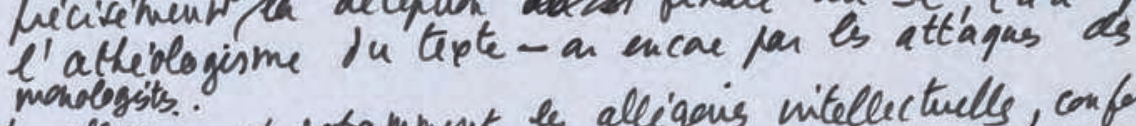

5- les alle'gons et ratamment es alligais nitellectrulls, confor me'-

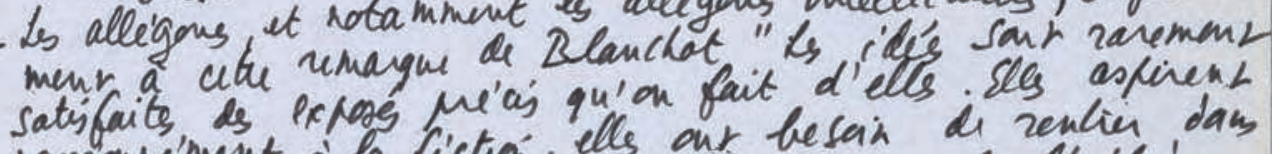

s/Cyran: $+559$ namonne'ment a' la fiction, ells our besain di renlien dam a monde". tonle zuthei daus le monde de l'idei', terte unscription du monde sas l'idée.

6 - Les conte-metaphas, cad ls mitaphas piss et de'velopiés a' la bethe. 'mitaphars qu' te réalisent. Kafka et la Me'camaphese (Marthe Robert); Cysano et Blenchat: marii de chafin (condamner a' une mert qu' n'ur faite que du chafuin de mowie "); recuecliei son dernier sonpis, de t'aime tanr qu' de te manferaj. "Metaptores q me' he réalisent par de hais mitamaptass" (Blanchat)

7 - La copie (l'imitatian), le double pupite de Barran et Pécuchet. In pert mithe ici la parodic de Sa' me me, e'criture de sai sur e'cu'ture de mai (nas en revas

Michelet Prif. 153 is cas en hitarie), le dialogue des(ruetem de dé ba. calisé.

8- les a racoluths: changemens de logigns an de et sem nicubrant_Subrepticement Ex: Denida. "Ka $2 a$.

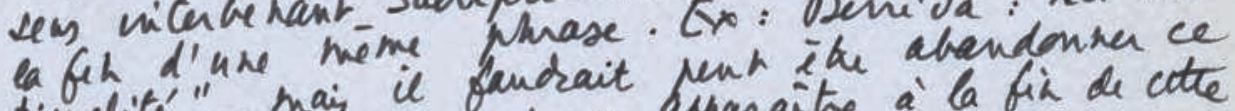

gramm tionalite' " mais is fandrait appraraite a' la fir de cite mot paur la zaiton qui

9. D'une menieir gériciale torts les connotatray (idé que

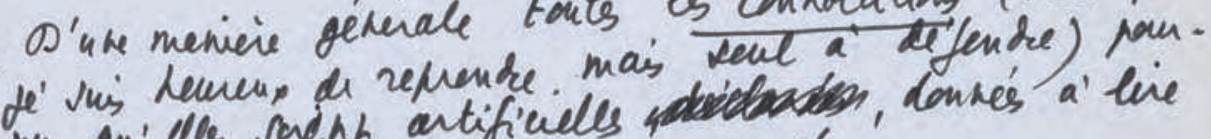

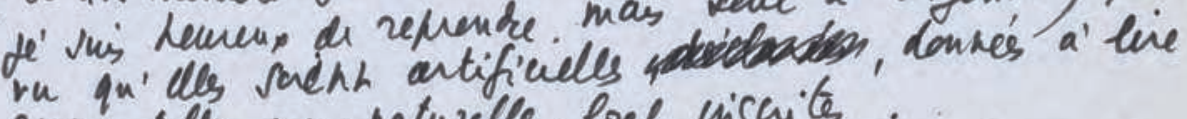
comme cills, non. naturells, bef viscites.

Clte life - qui'a'ur gn'an licut - montre qu'id ur poribe concerai it mime a proprammer une veste descriptien de l'écus ture paraframmetique.

Fig. 10

159 
pas de difficulté fondamentale au niveau des procédures (44 de découverte. Mais au niveau des procédures d'exposition? Dans quelle écriture allons-nous décrire le paragrammatisme? mono- ou polygrammatiste. Cela pose la question de la paragrammatique, ou discours concernant l'espace du langage, càd sur la littérature, càd discours critique.

\section{La Paragrammatique.}

- L'écriture paragrammatique : nécessité fondamentale : empêch le paragramme empêche la formation du Sé transcendantal, car à partir du moment où il y a paragrammatisme, il n'y a plus privilège et instance du Sé : tout paragrammatisme est a-théologique, il empêche la concrétion, même implicite, d'un Sé dernier qui «mettrait un terme rassurant au renvoi de signe à signe » (Derrida) ${ }^{33}$

- Reconnaître - et même louer - le paragrammatisme en usant d'une écriture monologique, scientifique, monovalente, théologique, c'est donc finalement renflouer le 1, l'indivis, le Sé transcendantal, la Présence, c'est coiffer libéralement le polygrammatisme par un monogrammatisme qui le « comprendrait». Ceci est évidemment intolérable.

- C'est pourtant ce que fait toute écriture d'écrivant, monologique par statut. Parler de littérature avec une écriture d'écrivant, de « savant », c'est faire peser plus lourdement que jamais sur la littérature, sur l'écriture, le tabou théologique du sens indivis. Cas pourtant général de la critique, surtout depuis qu'elle n'est plus protégée par la Rhétorique et n'ose pas encore réintégrer la littérature. On notera d' liaison entre ailleurs que l'écrivance (par abandon de la rhétorique) le technicisme est contemporaine de la technocratie, Les vrais suppôts et le logo- de la civilisation technique, ce ne sont pas les formalistes, centrisme (Derri- (les écrivains), ce sont les monologistes, les scientistes, les

da) $)^{34}$ écrivants. La seule force vraiment contestatrice, c'est le paragrammatisme : c'est ça, la Révolution.

- Le problème fondamental aujourd'hui n'est donc pas du tout d'élaborer une théorie critique, une théorie de la littérature, de la production littéraire, c'est de décider si une langue critique est possible et quelle elle doit être. Le problème de l'écriture, du statut linguistique de la « science » prime tous les autres. Quiconque veut parler de littérature, comment va-t-il écrire?

\footnotetext{
${ }^{33}$ J. Derrida, « De la grammatologie II », op. cit., p. 26.

${ }^{34}$ J. Derrida, « De la grammatologie I », op. cit.
} 
pas de difficulte' fondamentale an Mivean ds procidars (44 de dionverte. Mais an nivean de procidurs d'expontion? Dans quelle e'criture allan now dicine le paraframmatisine? mono-au plygrammatiste. Cela pese la gustar de la vara. grammatigue in dicams conceinant l'apace du lanjage, cad Sm ta titcírature, cad discons critigue.

4. La Paragrammatique.

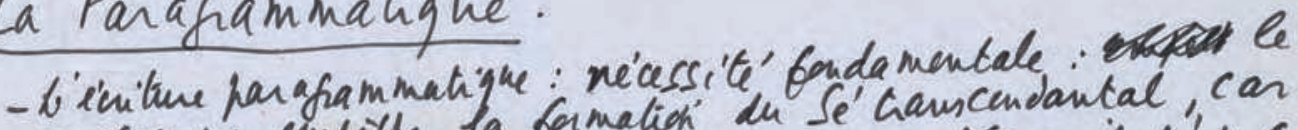
paragramine enficthe la formation' in Se' ciancendantal, car

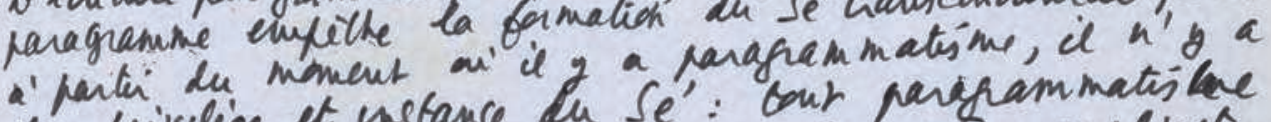
peus mivilize et unstance du Se': Cour paregrammatis loe ur a-che'ologigne, il emfich la conchtha, mime implicite, d' un Se' derhie qun " vethait un lis me rasserant au zen ra' de Signe a'signe "(Denida)

- Reconnaitre - et méme bover - le paraframmatis'me en cesant d'une e'cutare monologique, scientifi'gue, monoralente, thiologique, c'ar donc finalement zonflerer le 1, l'mi.

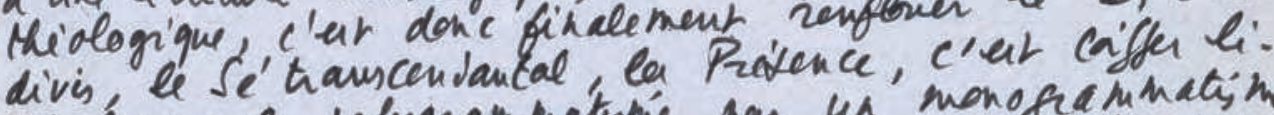
biralencur el polygrammatsme pan un monofiamuatime qui \& "ompentrait". Ceci us e'cidemment in colizable.

- C'err poutant a que fait tarte e'ci'ture d'écrivant, monolo. giqus per statut. Pailer de litcirature anec use icu'ture d'écivant, de "Savant", c'ar faire peser ples lourde mons que Lamais Sm la littirature, sin l'ecriture, le cabon thido. gique du ters undivis. Cas pantanr géreíal de la culigue,

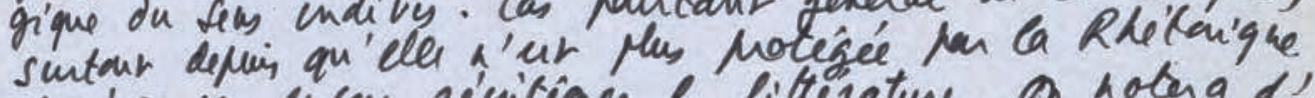
It $n^{\prime} x$ pas encou récicigrer la litierature. On notera d'

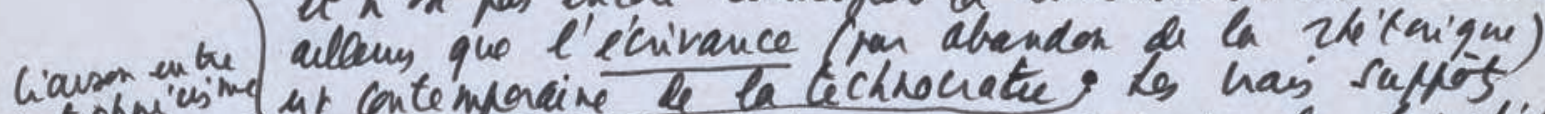

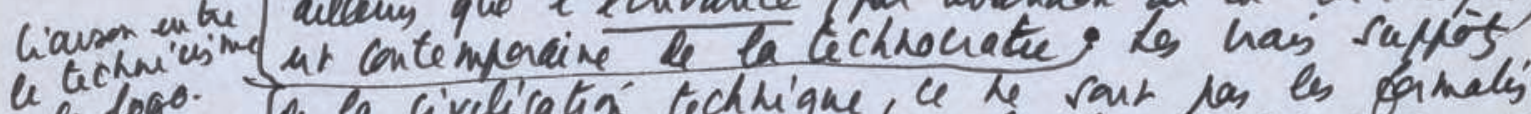

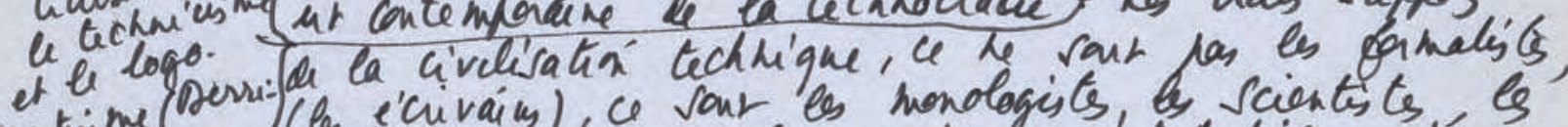

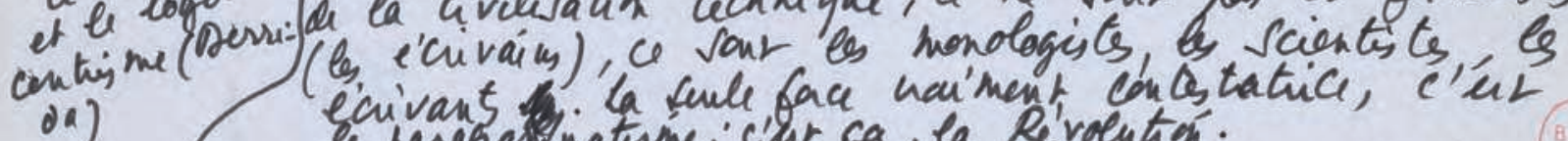
e rangramitinatesme: c'ar ca, la Révolutón.

- Le problime ofordemental acyand'hie n'ur done pas da tonk d'ilebarer ure théove cu'tique, use théne de la littérature, a la moductañ littéraire, c'ur de dicider $r$ un lengue critique ur posibe et quelle lle dois ithe. Le poblime de l'éciture, du statut leviguitique of ea "science" prime tous bs auts. quiconque veut perber de litletature, comment ra. Ail e'cive?

Fig. 11

161 
- [Ceci : a été mon problème dès le début : problème informulable, incommunicable puisqu'il faut précisément disposer d'une langue pour le communiquer.]

- Ce problème : trouver, pour énoncer la littérature, une langue isomorphe à cette littérature, càd un discours qui évite le monologisme, l'interdit scientiste et théologique.

a) Julia Kristeva, ne voit qu'une solution : recours au formalisme des mathématiques et des méta-mathématiques : « liberté des signes : échapper aux contraintes de la logique élaborée à partir de la phrase indo-européenne (sujet-prédicat) ${ }^{35}$.

Peut-être. Mais je crois plutôt : ce discours sera connoté immédiatement comme «mathématique », «formaliste », « scientifique». Le barrage ne sera que reculé.

b) Inventer une écriture critique qui soit elle-même paragrammatique? Seule lueur vers laquelle il faut aller. Quelles voies, quels actes, quels modes d'inscription ? On ne sait encore ; par ex : voies de truquage subreptice d'un monologisme apparent; ou encore critique-roman, à forme quasi monstrueuse

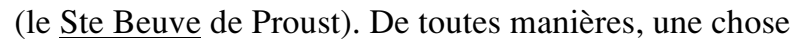
sûre marque le départ de cette invention fondamentale : rejet, pour parler de la litt, de l'écriture des écrivants : il faut écrire : ce qu'on dit de l'écriture doit être écrit; et tout ce qui s'est parlé ici et se parlera ici - précisons le nettement pour terminer cette introduction - doit être reçu comme relevant des «procédures de découvertes » et non des procédures d'exposition ».

\footnotetext{
${ }^{35}$ Voir J. Kristeva, « Pour une sémiologie des paragrammes », art. cit.
} 
- Cleci: a ele' mon problime dis 'L dilur moblime hi. (45 desposer a'ure lavfus pour le comimunigner.]

- A problime: tonver, pour inoncer la litlérature, une las. gue issomale a'cette littirature, cad uh discaus qui e'vite le monologisme, l'vicudit' scuentiste et thiologique.

a) Jubia Kriteva, he vair qu'une Soluth: recans an Gormalisme os mathimatiqus et ds mi'ta. Mathe'matiqus. "lifute' des signs. e'chaprer aup containts \& la logique elaborée a' parter' de la phrase indo-enrteik.

Peur (sajet-Midicat). Maij re reutot: 4 dicaus tera connoa' immidialement comme "mathimatique", "bamalite "scieitifique". \& hanafe he tera que reculé.

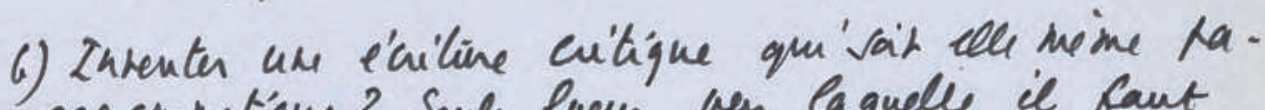
ragrammatique?. Seule lueur wes laquelle il fant der queles rais, queb acts, queb mods d'in'scinp. tion? on he sait encali par ex: rais de hu. quage subreftice I'un monologime aprarent, on encae critique - $20 \mathrm{man}$, a'farme quasi monsernerese (le Se Beuve de Ront). Re tants maneis, ure Chese sine marque a dipart de cete envention Gendamen. tale: rejet, pour parler \& la libt, de l'e'citure es e'crivan5: is faut e'criréce qu'on dit de

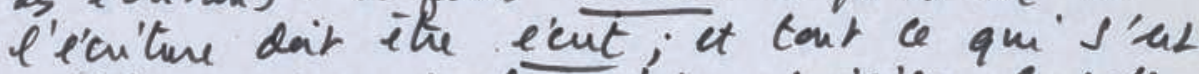
parle'ici et se harlera íci-me'aisas $b$ relte.

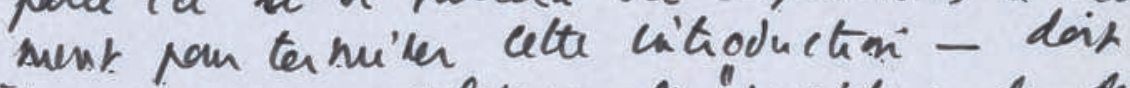
ithe refu comme velevans "proce'durs de dicon. rals "et non defacidurs d'expoxitin'." 


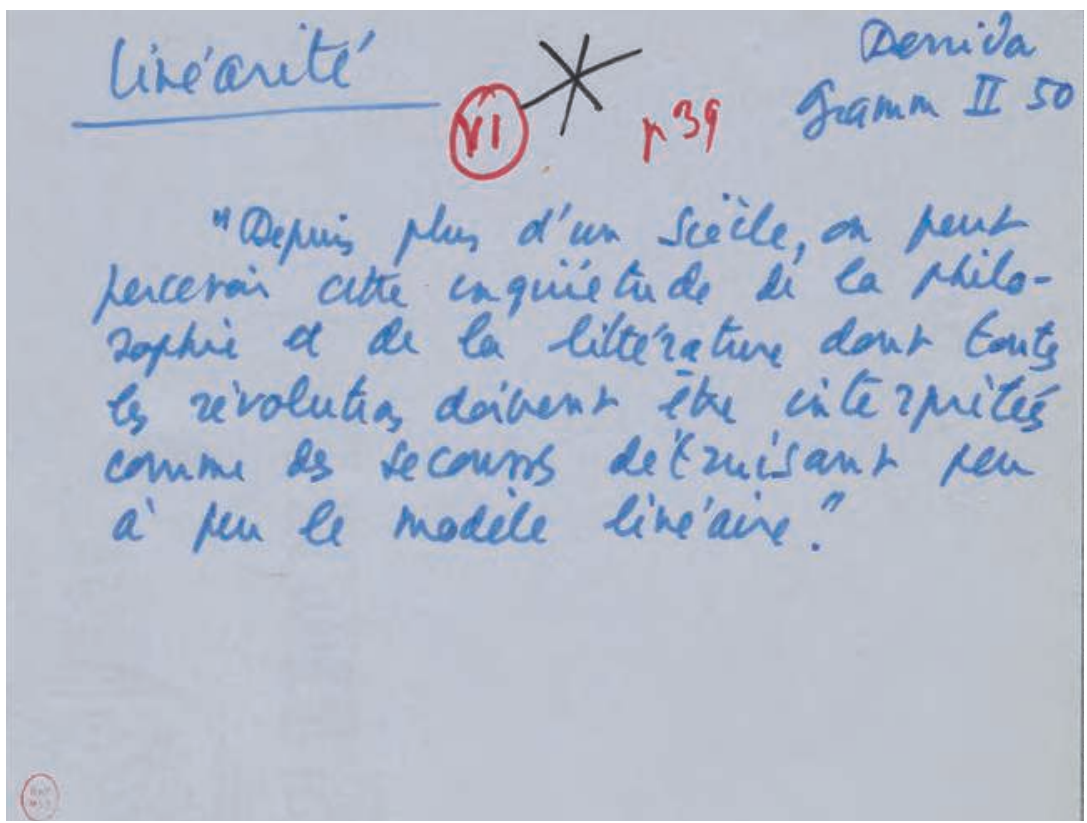

\begin{tabular}{lll}
\hline Linéarité & $\mathcal{K}_{\text {p } 39}$ & Derrida \\
& Gramm II 50
\end{tabular}

«Depuis plus d'un siècle, on peut percevoir cette inquiétude de la philosophie et de la littérature dont toutes les révolutions doivent être interprétées comme des secousses détruisant peu à peu le modèle linéaire. »

Fig. 13 